\title{
Geodesic Equations on Diffeomorphism Groups ${ }^{\star}$
}

\author{
Cornelia VIZMAN
}

Department of Mathematics, West University of Timisoara, Romania

E-mail:vizman@math.uvt.ro

Received November 13, 2007, in final form March 01, 2008; Published online March 11, 2008

Original article is available at http://www.emis.de/journals/SIGMA/2008/030/

\begin{abstract}
We bring together those systems of hydrodynamical type that can be written as geodesic equations on diffeomorphism groups or on extensions of diffeomorphism groups with right invariant $L^{2}$ or $H^{1}$ metrics. We present their formal derivation starting from Euler's equation, the first order equation satisfied by the right logarithmic derivative of a geodesic in Lie groups with right invariant metrics.
\end{abstract}

Key words: Euler's equation; diffeomorphism group; group extension; geodesic equation

2000 Mathematics Subject Classification: 58D05; 35Q35

A fluid moves to get out of its own way as efficiently as possible.

Joe Monaghan

\section{Introduction}

Some conservative systems of hydrodynamical type can be written as geodesic equations on the group of diffeomorphisms or the group of volume preserving diffeomorphisms of a Riemannian manifold, as well as on extensions of these groups. Considering right invariant $L^{2}$ or $H^{1}$ metrics on these infinite dimensional Lie groups, the following geodesic equations can be obtained: the Euler equation of motion of a perfect fluid [2, 10], the averaged Euler equation [31, 50], the equations of ideal magneto-hydrodynamics [54, 32], the Burgers inviscid equation [7], the template matching equation [18, 55], the Korteweg-de Vries equation [44], the Camassa-Holm shallow water equation $[8,38,29]$, the higher dimensional Camassa-Holm equation (also called EPDiff or averaged template matching equation) [20], the superconductivity equation [49], the equations of motion of a charged ideal fluid [57], of an ideal fluid in Yang-Mills field [14] and of a stratified fluid in Boussinesq approximation [61, 58].

For a Lie group $G$ with right invariant metric, the geodesic equation written for the right logarithmic derivative $u$ of the geodesic is a first order equation on the Lie algebra $\mathfrak{g}$, called the Euler equation. Denoting by $\operatorname{ad}(u)^{\top}$ the adjoint of $\operatorname{ad}(u)$ with respect to the scalar product on $\mathfrak{g}$ given by the metric, Euler's equation can be written as $\frac{d}{d t} u=-\operatorname{ad}(u)^{\top} u$. In this survey type article we do the formal derivation of all the equations of hydrodynamical type mentioned above, starting from this equation.

By writing such partial differential equations as geodesic equations on diffeomorphism groups, there are various properties one can obtain using the Riemannian geometry of right invariant metrics on these diffeomorphism groups. We will not focus on them in this paper, but we list some of them below, with some of the references.

For some of these equations smoothness of the geodesic spray on the group implies local wellposedness of the Cauchy problem as well as smooth dependence on the initial data. This applies

${ }^{\star}$ This paper is a contribution to the Proceedings of the Seventh International Conference "Symmetry in Nonlinear Mathematical Physics" (June 24-30, 2007, Kyiv, Ukraine). The full collection is available at http://www.emis.de/journals/SIGMA/symmetry2007.html 
for the following right invariant Riemannian metrics: $L^{2}$ metric on the group of volume preserving diffeomorphisms [10], $H^{1}$ metric on the group of volume preserving diffeomorphisms on a boundary free manifold [50], on a manifold with Dirichlet boundary conditions [31, 51] and with Neumann or mixt boundary conditions [51,13], $H^{1}$ metric on the group of diffeomorphisms of the circle [50, 29] and on the Bott-Virasoro group [9], and $H^{1}$ metric on the group of diffeomorphisms on a higher dimensional manifold [15].

There are also results on the sectional curvature (with information on the Lagrangian stability) $[2,42,48,39,34,46,56,17,63,62,57]$, on the existence of conjugate points [37, 40] and minimal geodesics [6], on the finiteness of the diameter [52, 53, 11], on the vanishing of geodesic distance [33], as well as on the Riemannian geometry of subgroups of diffeomorphisms as a submanifold of the full diffeomorphism group [36, 4, 26, 55].

\section{Euler's equation}

Given a regular Fréchet-Lie group in the sense of Kriegl-Michor [28], and a (positive definite) scalar product $\langle\rangle:, \mathfrak{g} \times \mathfrak{g} \rightarrow \mathbb{R}$ on the Lie algebra $\mathfrak{g}$, we can define a right invariant metric on $G$ by $g_{x}(\xi, \eta)=\left\langle\xi x^{-1}, \eta x^{-1}\right\rangle$ for $\xi, \eta \in T_{x} G$. The energy functional of a smooth curve $c: I=[a, b] \rightarrow G$ is defined by

$$
E(c)=\frac{1}{2} \int_{a}^{b} g_{c(t)}\left(c^{\prime}(t), c^{\prime}(t)\right) d t=\frac{1}{2} \int_{a}^{b}\left\langle\delta^{r} c(t), \delta^{r} c(t)\right\rangle d t
$$

where $\delta^{r}$ denotes the right logarithmic derivative (angular velocity) on the Lie group $G$, i.e. $\delta^{r} c(t)=c^{\prime}(t) c(t)^{-1} \in \mathfrak{g}$. We assume the adjoint of $\operatorname{ad}(X)$ with respect to $\langle$,$\rangle exists for all$ $X \in \mathfrak{g}$ and we denote it by $\operatorname{ad}(X)^{\top}$, i.e.

$$
\left\langle\operatorname{ad}(X)^{\top} Y, Z\right\rangle=\langle Y,[X, Z]\rangle, \quad \forall X, Y, Z \in \mathfrak{g} .
$$

The corresponding notation in $[3]$ is $B(X, Y)=\operatorname{ad}(Y)^{\top} X$ for the bilinear map $B: \mathfrak{g} \times \mathfrak{g} \rightarrow \mathfrak{g}$.

Theorem 1. The curve $c:[a, b] \rightarrow G$ is a geodesic for the right invariant metric $g$ on $G$ if and only if its right logarithmic derivative $u=\delta^{r} c:[a, b] \rightarrow \mathfrak{g}$ satisfies the Euler equation:

$$
\frac{d}{d t} u=-\operatorname{ad}(u)^{\top} u
$$

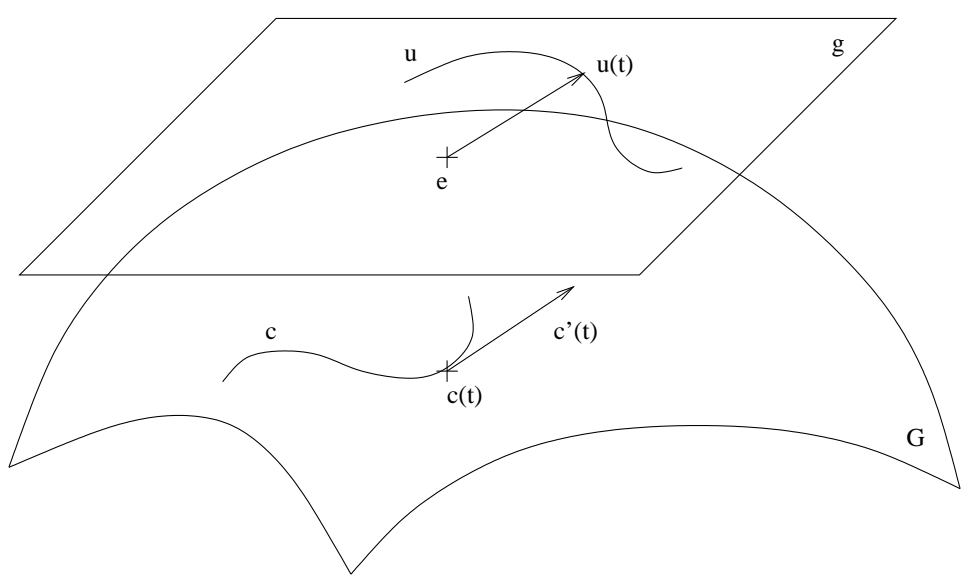


Proof. We denote the given curve by $c_{0}$ and its logarithmic derivative by $u_{0}$. For any variation with fixed endpoints $c(t, s) \in G, t \in[a, b], s \in(-\varepsilon, \varepsilon)$ of the given curve $c_{0}$, we define $u=$ $\left(\partial_{t} c\right) c^{-1}$ and $v=\left(\partial_{s} c\right) c^{-1}$. In particular $u(\cdot, 0)=u_{0}$, and we denote $v(\cdot, 0)$ by $v_{0}$.

Following [35] we show first that

$$
\partial_{t} v-\partial_{s} u=[u, v]
$$

For each $h \in G$ we consider the map $F_{h}(t, s)=(t, s, c(t, s) h)$ for $t \in[a, b]$ and $s \in(-\varepsilon, \varepsilon)$. The bracket of the following two vector fields on $[a, b] \times(-\varepsilon, \varepsilon) \times G$ vanishes:

$$
(t, s, g) \mapsto \partial_{t}+u(t, s) g, \quad(t, s, g) \mapsto \partial_{s}+v(t, s) g .
$$

The reason is they correspond under the mappings $F_{h}, h \in G$, to the vector fields $\partial_{t}$ and $\partial_{s}$ on $[a, b] \times(-\varepsilon, \varepsilon)$ (with vanishing bracket). Hence $0=\left[\partial_{t}+u g, \partial_{s}+v g\right]=\left(\partial_{t} v\right) g-\left(\partial_{s} u\right) g-[u, v] g$, because the bracket of right invariant vector fields corresponds to the opposite bracket on the Lie algebra $\mathfrak{g}$, so the claim (2.2) follows.

As in [34] we compute the derivative of $E(c)=\frac{1}{2} \int_{a}^{b}\langle u, u\rangle d t$ with respect to $s$, using the fact that $v(a, s)=v(b, s)=0$.

$$
\partial_{s} E(c)=\int_{a}^{b}\left\langle\partial_{s} u, u\right\rangle d t \stackrel{(2.2)}{=} \int_{a}^{b}\left\langle\partial_{t} v-[u, v], u\right\rangle d t=-\int_{a}^{b}\left\langle v, \partial_{t} u+\operatorname{ad}(u)^{\top} u\right\rangle d t .
$$

The curve $c_{0}$ in $G$ is a geodesic if and only if this derivative vanishes at $s=0$ for all variations $c$ of $c_{0}$, hence for all $v_{0}:[a, b] \rightarrow \mathfrak{g}$. This is equivalent to $\frac{d}{d t} u_{0}=-\operatorname{ad}\left(u_{0}\right)^{\top} u_{0}$.

The Euler equation for a left invariant metric on a Lie group is $\frac{d}{d t} u=\operatorname{ad}(u)^{\top} u$. In the case $G=S O(3)$ one obtains the equations of the rigid body.

Denoting by $\left(\right.$, ) the pairing between $\mathfrak{g}^{*}$ and $\mathfrak{g}$, the inertia operator [3] is defined by

$$
A: \mathfrak{g} \rightarrow \mathfrak{g}^{*}, \quad A(X)=\langle X, \cdot\rangle, \quad \text { i.e. } \quad(A(X), Y)=\langle X, Y\rangle, \quad \forall X, Y \in \mathfrak{g} .
$$

It is injective, but not necessarily surjective for infinite dimensional $\mathfrak{g}$. The image of $A$ is called the regular part of the dual and is denoted by $\mathfrak{g}_{\mathrm{reg}}^{*}$.

Let $\mathrm{ad}^{*}$ be the coadjoint action of $\mathfrak{g}$ on $\mathfrak{g}^{*}$ given by $\left(\operatorname{ad}^{*}(X) m, Y\right)=(m,-\operatorname{ad}(X) Y)$, for $m \in \mathfrak{g}^{*}$. The inertia operator relates $\operatorname{ad}(X)^{\top}$ to the opposite of the coadjoint action of $X$, i.e.

$$
\operatorname{ad}^{*}(X) A(Y)=-A\left(\operatorname{ad}(X)^{\top} Y\right) .
$$

Hence the inertia operator transforms the Euler equation (2.1) into an equation for $m=A(u)$ :

$$
\frac{d}{d t} m=\operatorname{ad}^{*}(u) m
$$

result known also as the second Euler theorem.

First Euler theorem states that the solution of $(2.4)$ with $m(a)=m_{0}$ is

$$
m(t)=\operatorname{Ad}^{*}(c(t)) m_{0},
$$

where $u=\delta^{r} c$ and $c(a)=e$. Indeed, $\frac{d}{d t} m=\operatorname{ad}^{*}\left(\delta^{r} c\right) \operatorname{Ad}^{*}(c) m_{0}=\operatorname{ad}^{*}(u) m$.

Remark 1. Equation (2.4) is a Hamiltonian equation on $\mathfrak{g}^{*}$ with the canonical Poisson bracket

$$
\{f, g\}(m)=\left(m,\left[\frac{\delta f}{\delta m}, \frac{\delta g}{\delta m}\right]\right), \quad f, g \in C^{\infty}\left(\mathfrak{g}^{*}\right)
$$

and the Hamiltonian function $h \in C^{\infty}\left(\mathfrak{g}^{*}\right), h(m)=\frac{1}{2}\left(m, A^{-1} m\right)=\frac{1}{2}(m, u)$. 
Remark 2. The Euler-Lagrange equation for a right invariant Lagrangian $L: T G \rightarrow \mathbb{R}$ with value $l: \mathfrak{g} \rightarrow \mathbb{R}$ at the identity is:

$$
\frac{d}{d t} \frac{\delta l}{\delta u}=\operatorname{ad}^{*}(u) \frac{\delta l}{\delta u},
$$

also called the right Euler-Poincaré equation [47, 30]. The Hamiltonian form (2.4) of Euler's equation is obtained for $l(u)=\frac{1}{2}\langle u, u\rangle$ since the functional derivative $\frac{\delta l}{\delta u}$ is $A(u)$ in this case.

\section{Ideal hydrodynamics}

Let $G=\operatorname{Diff}_{\mu}(M)$ be the regular Fréchet Lie group of volume preserving diffeomorphisms of a compact Riemannian manifold $(M, g)$ with induced volume form $\mu$. Its Lie algebra is $\mathfrak{g}=\mathfrak{X}_{\mu}(M)$, the Lie algebra of divergence free vector fields, with Lie bracket the opposite of the usual bracket of vector fields $\operatorname{ad}(X) Y=-[X, Y]$. We consider the right invariant metric on $G$ given by the $L^{2}$ scalar product on vector fields

$$
\langle X, Y\rangle=\int_{M} g(X, Y) \mu .
$$

In the $L^{2}$ orthogonal decomposition $\mathfrak{X}(M)=\mathfrak{X}_{\mu}(M) \oplus \operatorname{grad}\left(C^{\infty}(M)\right)$, we denote by $P$ the projection on $\mathfrak{X}_{\mu}(M)$. The adjoint of $\operatorname{ad}(X)$ is $\operatorname{ad}(X)^{\top} Y=P\left(\nabla_{X} Y+(\nabla X)^{\top} Y\right)$ where $\nabla$ denotes the Levi-Civita covariant derivative. Indeed,

$$
\begin{aligned}
\left\langle\operatorname{ad}(X)^{\top} Y, Z\right\rangle & =\int_{M} g(Y,[Z, X]) \mu=\int_{M} g\left(Y, \nabla_{Z} X-\nabla_{X} Z\right) \mu \\
& =\int_{M} g\left((\nabla X)^{\top} Y, Z\right) \mu+\int_{M} g\left(\nabla_{X} Y, Z\right) \mu=\left\langle P\left(\nabla_{X} Y+(\nabla X)^{\top} Y\right), Z\right\rangle,
\end{aligned}
$$

with $(\nabla X)^{\top}$ denoting the adjoint of the $(1,1)$-tensor $\nabla X$ relative to the metric: $g\left(\nabla_{Z} X, Y\right)=$ $g\left(Z,(\nabla X)^{\top} Y\right)$. In particular $\operatorname{ad}(X)^{\top} X=P\left(\nabla_{X} X\right)=\nabla_{X} X+\operatorname{grad} p$, with $p$ the smooth function uniquely defined up to a constant by $\Delta p=\operatorname{div}\left(\nabla_{X} X\right)$. Now Theorem 1 assures that the geodesic equation in $\operatorname{Diff}_{\mu}(M)$, in terms of the right logarithmic derivative $u$ of the geodesic, is Euler's equation for ideal flow with velocity $u$ and pressure $p[41,2,10]$ :

$$
\partial_{t} u=-\nabla_{u} u-\operatorname{grad} p, \quad \operatorname{div} u=0 .
$$

The geodesic equation (3.2) written for the vorticity 2 -form $\omega=d u^{b}, b$ denoting the inverse of the Riemannian lift $\sharp$ and $L$ the Lie derivative, is

$$
\partial_{t} \omega=-L_{u} \omega
$$

because $\left(\nabla_{u} u\right)^{b}=L_{u} u^{b}-\frac{1}{2} d(g(u, u))$ and $(\operatorname{grad} p)^{b}=d p$.

\section{Burgers equation}

Let $G=\operatorname{Diff}\left(S^{1}\right)$ be the group of orientation preserving diffeomorphisms of the circle and $\mathfrak{g}=\mathfrak{X}\left(S^{1}\right)$ the Lie algebra of vector fields. The Lie bracket is $[X, Y]=X^{\prime} Y-X Y^{\prime}$, the negative of the usual bracket on vector fields (vector fields on the circle are identified here with their coefficient functions in $C^{\infty}\left(S^{1}\right)$ ). We consider the right invariant metric on $G$ given by the $L^{2}$ scalar product $\langle X, Y\rangle=\int_{S^{1}} X Y d x$ on $\mathfrak{g}$. The adjoint of $\operatorname{ad}(X)$ is $\operatorname{ad}(X)^{\top} Y=2 X^{\prime} Y+X Y^{\prime}$, because:

$$
\left\langle\operatorname{ad}(X)^{\top} Y, Z\right\rangle=\int_{S^{1}} Y\left(X^{\prime} Z-X Z^{\prime}\right) d x=\int_{S^{1}}\left(X^{\prime} Y+(X Y)^{\prime}\right) Z d x=\left\langle 2 X^{\prime} Y+X Y^{\prime}, Z\right\rangle .
$$


It follows from Theorem 1 that the geodesic equation on $\operatorname{Diff}\left(S^{1}\right)$ in terms of the right logarithmic derivative $u: I \rightarrow C^{\infty}\left(S^{1}\right)$ is Burgers inviscid equation [7]:

$$
\partial_{t} u=-3 u u^{\prime}
$$

The higher dimensional Burgers equation is the template matching equation, used for comparing images via a deformation induced distance. It is the geodesic equation on $\operatorname{Diff}(M)$, the diffeomorphism group of a compact Riemannian manifold $(M, g)$, for the right invariant $L^{2}$ metric $[18,55]$ :

$$
\partial_{t} u=-\nabla_{u} u-(\operatorname{div} u) u-\frac{1}{2} \operatorname{grad} g(u, u) .
$$

Indeed,

$$
\operatorname{ad}(X)^{\top}=(\operatorname{div} X) 1+\nabla_{X}+(\nabla X)^{\top}, \quad \forall X \in \mathfrak{X}(M),
$$

because as in Section 3 we compute $\left\langle\operatorname{ad}(X)^{\top} Y, Z\right\rangle=\int_{M} g\left((\nabla X)^{\top} Y, Z\right) \mu+\int_{M} g\left(\nabla_{X} Y, Z\right) \mu-$ $\int_{M} L_{X} g(Y, Z) \mu=\left\langle(\nabla X)^{\top} Y+\nabla_{X} Y+(\operatorname{div} X) Y, Z\right\rangle$ for all vector fields $X, Y, Z$ on $M$.

In particular for $M=S^{1}$ and $u$ a curve in $\mathfrak{X}\left(S^{1}\right)$, identified with $C^{\infty}\left(S^{1}\right)$, div $u=u^{\prime}$ and $g(u, u)=u^{2}$, so each of the three terms in the right hand side of (4.2) is $-u u^{\prime}$ and we recover Burgers equation (4.1).

\section{$5 \quad$ Abelian extensions}

A bilinear skew-symmetric map $\omega: \mathfrak{g} \times \mathfrak{g} \rightarrow V$ is a 2-cocycle on the Lie algebra $\mathfrak{g}$ with values in the $\mathfrak{g}$-module $V$ if it satisfies the condition

$$
\sum_{\text {cycl }} \omega\left(\left[X_{1}, X_{2}\right], X_{3}\right)=\sum_{\text {cycl }} b\left(X_{1}\right) \omega\left(X_{2}, X_{3}\right), \quad X_{1}, X_{2}, X_{3} \in \mathfrak{g},
$$

where $b: \mathfrak{g} \rightarrow L(V)$ denotes the Lie algebra action on $V$. It determines an Abelian Lie algebra extension $\hat{\mathfrak{g}}:=V \rtimes_{\omega} \mathfrak{g}$ of $\mathfrak{g}$ by the $\mathfrak{g}$-module $V$ with Lie bracket

$$
\left[\left(v_{1}, X_{1}\right),\left(v_{2}, X_{2}\right)\right]=\left(b\left(X_{1}\right) v_{2}-b\left(X_{2}\right) v_{1}+\omega\left(X_{1}, X_{2}\right),\left[X_{1}, X_{2}\right]\right) .
$$

There is a 1-1 correspondence between the second Lie algebra cohomology group $H^{2}(\mathfrak{g}, V)$ and equivalence classes of Abelian Lie algebra extensions $0 \rightarrow V \rightarrow \hat{\mathfrak{g}} \rightarrow \mathfrak{g} \rightarrow 0$.

When $G$ is infinite dimensional, the two obstructions for the integrability of such an Abelian Lie algebra extension to a Lie group extension of the connected Lie group $G$ involve $\pi_{1}(G)$ and $\pi_{2}(G)$ [43]. The Lie algebra 2-cocycle $\omega$ is integrable if

- the period group $\Pi_{\omega} \subset V$ (the group of spherical periods of the equivariant $V$-valued 2 -form on $G$ defined by $\omega$ ) is discrete and

- the flux homomorphism $F_{\omega}: \pi_{1}(G) \rightarrow H^{1}(\mathfrak{g}, V)$ vanishes.

Then for any discrete subgroup $\Gamma$ of the subspace of $\mathfrak{g}$-invariant elements of $V$ with $\Gamma \supseteq \Pi_{\omega}$, there is an Abelian Lie group extension $1 \rightarrow T \rightarrow \hat{G} \rightarrow G \rightarrow 1$ of $G$ by $T=V / \Gamma$.

There are two special cases:

1. Semidirect product: $\hat{\mathfrak{g}}=V \rtimes \mathfrak{g}$, obtained when $\omega=0$.

An example is the semidirect product $\mathfrak{g}^{*} \rtimes G$ for the coadjoint $G$-action on $\mathfrak{g}^{*}$, called the magnetic extension in [3]. It has the Lie algebra $\mathfrak{g}^{*} \rtimes \mathfrak{g}$, a semidirect product for the coadjoint $\mathfrak{g}$-action $b=\operatorname{ad}^{*}$ on $\mathfrak{g}^{*}$. 
2. Central extension: $\hat{\mathfrak{g}}=V \times_{\omega} \mathfrak{g}$, obtained when $b=0$.

An example is the Virasoro algebra $\mathbb{R} \times{ }_{\omega} \mathfrak{X}\left(S^{1}\right)$, a central extension of the Lie algebra of vector fields on the circle given by the Virasoro cocycle $\omega(X, Y)=\int_{S^{1}}\left(X^{\prime} Y^{\prime \prime}-X^{\prime \prime} Y^{\prime}\right) d x$. It has a corresponding Lie group extension of the group $\operatorname{Diff}\left(S^{1}\right)$ of orientation preserving diffeomorphisms of the circle, defined by the Bott group cocycle:

$$
c(\varphi, \psi)=\int_{S^{1}} \log \left(\varphi^{\prime} \circ \psi\right) d \log \psi^{\prime}, \quad \varphi, \psi \in \operatorname{Diff}\left(S^{1}\right) .
$$

An example of a general Abelian Lie algebra extension is $C^{\infty}(M) \rtimes_{\omega} \mathfrak{X}(M)$, the Abelian extension of the Lie algebra of vector fields on the manifold $M$ with the opposite bracket by the natural module of smooth functions on $M$, the Lie algebra action being $b(X) f=-L_{X} f$. The cocycle $\omega: \mathfrak{X}(M) \times \mathfrak{X}(M) \rightarrow C^{\infty}(M)$ is given by a closed differential 2-form $\eta$ on $M$. If $\eta$ is an integral form, then there is a principal circle bundle $P$ over $M$ with curvature $\eta$. In this case the group of equivariant automorphisms of $P$ is a Lie group extension integrating the Lie algebra cocycle $\omega$ :

$$
1 \rightarrow C^{\infty}(M, \mathbb{T}) \rightarrow \operatorname{Diff}(P)^{\mathbb{T}} \rightarrow \operatorname{Diff}(M)_{[P]} \rightarrow 1
$$

Here $C^{\infty}(M, \mathbb{T})$ is the gauge group of $P$ and $\operatorname{Diff}(M)_{[P]}$ is the group of diffeomorphisms of $M$ preserving the bundle class $[P]$ under pullbacks (group having the same identity component as $\operatorname{Diff}(M))$.

\section{Geodesic equations on Abelian extensions}

Following [57] we write down the geodesic equations on an Abelian Lie group extension $\hat{G}$ of $G$ with respect to the right invariant metric defined with the scalar product

$$
\left\langle\left(v_{1}, X_{1}\right),\left(v_{2}, X_{2}\right)\right\rangle_{\hat{\mathfrak{g}}}=\left\langle v_{1}, v_{2}\right\rangle_{V}+\left\langle X_{1}, X_{2}\right\rangle_{\mathfrak{g}}
$$

on its Lie algebra $\hat{\mathfrak{g}}=V \rtimes_{\omega} \mathfrak{g}$. Here $\langle,\rangle_{\mathfrak{g}}$ and $\langle,\rangle_{V}$ are scalar products on $\mathfrak{g}$ and $V$. We have to assume the existence of the following maps: the adjoint $\operatorname{ad}(X)^{\top}: \mathfrak{g} \rightarrow \mathfrak{g}$ and the adjoint $b(X)^{\top}: V \rightarrow V$ for any $X \in \mathfrak{g}$, the linear map $h: V \rightarrow L_{\text {skew }}(\mathfrak{g})$ taking values in the space of skew-adjoint operators on $\mathfrak{g}$, defined by

$$
\left\langle h(v) X_{1}, X_{2}\right\rangle_{\mathfrak{g}}=\left\langle\omega\left(X_{1}, X_{2}\right), v\right\rangle_{V}
$$

and the bilinear map $l: V \times V \rightarrow \mathfrak{g}$, defined by

$$
\left\langle l\left(v_{1}, v_{2}\right), X\right\rangle_{\mathfrak{g}}=\left\langle b(X) v_{1}, v_{2}\right\rangle_{V}
$$

The diamond operation $\diamond: V \times V^{*} \rightarrow \mathfrak{g}$ in [19] corresponds to our map $l$ via $\langle,\rangle_{V}$.

Proposition 1. The geodesic equation on the Abelian extension $\hat{G}$ for the right invariant metric defined by the scalar product (6.1) on $\hat{\mathfrak{g}}$, written for the right logarithmic derivative $(f, u)$, i.e. for curves $u$ in $\mathfrak{g}$ and $f$ in $V$, is

$$
\begin{aligned}
& \frac{d}{d t} u=-\operatorname{ad}(u)^{\top} u-h(f) u+l(f, f), \\
& \frac{d}{d t} f=-b(u)^{\top} f .
\end{aligned}
$$


Proof. We compute the adjoint of $\operatorname{ad}(v, X)$ in $V \rtimes_{\omega} \mathfrak{g}$ w.r.t. the scalar product (6.1)

$$
\begin{aligned}
& \left\langle\operatorname{ad}\left(v_{1}, X_{1}\right)^{\top}\left(v_{2}, X_{2}\right),\left(v_{3}, X_{3}\right)\right\rangle_{\hat{\mathfrak{g}}}=\left\langle\left(v_{2}, X_{2}\right),\left(b\left(X_{1}\right) v_{3}-b\left(X_{3}\right) v_{1}+\omega\left(X_{1}, X_{3}\right),\left[X_{1}, X_{3}\right]\right\rangle_{\hat{\mathfrak{g}}}\right. \\
& \quad=\left\langle v_{2}, b\left(X_{1}\right) v_{3}\right\rangle_{V}+\left\langle X_{2},\left[X_{1}, X_{3}\right]\right\rangle_{\mathfrak{g}}+\left\langle v_{2}, \omega\left(X_{1}, X_{3}\right)\right\rangle_{V}-\left\langle v_{2}, b\left(X_{3}\right) v_{1}\right\rangle_{V} \\
& \quad=\left\langle\left(b\left(X_{1}\right)^{\top} v_{2}, \operatorname{ad}\left(X_{1}\right)^{\top} X_{2}+h\left(v_{2}\right) X_{1}-l\left(v_{1}, v_{2}\right)\right),\left(v_{3}, X_{3}\right)\right\rangle_{\hat{\mathfrak{g}}} .
\end{aligned}
$$

The result follows now from Euler's equation (2.1).

Remark 3. When the scalar product on $V$ is g-invariant, i.e. $\left\langle b(X) v_{1}, v_{2}\right\rangle_{V}+\left\langle v_{1}, b(X) v_{2}\right\rangle_{V}=0$, then $l$ is skew-symmetric and the geodesic equation becomes

$$
\begin{aligned}
\frac{d}{d t} u & =-\operatorname{ad}(u)^{\top} u-h(f) u, \\
\frac{d}{d t} f & =b(u) f .
\end{aligned}
$$

\section{Geodesic equations on semidirect products}

A special case of Proposition 1, obtained for $\omega=0$, is:

Corollary 1. The geodesic equation on the semidirect product Lie group $V \rtimes G$ for the right invariant metric defined by the scalar product (6.1), written for the curve $(f, u)$ in $V \rtimes \mathfrak{g}$, is

$$
\begin{aligned}
& \frac{d}{d t} u=-\operatorname{ad}(u)^{\top} u+l(f, f), \\
& \frac{d}{d t} f=-b(u)^{\top} f .
\end{aligned}
$$

It reduces to

$$
\begin{aligned}
& \frac{d}{d t} u=-\operatorname{ad}(u)^{\top} u, \\
& \frac{d}{d t} f=b(u) f
\end{aligned}
$$

when the scalar product on $V$ is $\mathfrak{g}$-invariant.

\section{Passive scalar motion}

The geodesic equation on the semidirect product $C^{\infty}(M) \rtimes \operatorname{Diff}_{\mu}(M)$ with $L^{2}$ right invariant metric, written for the right logarithmic derivative $(f, u): I \rightarrow C^{\infty}(M) \rtimes \mathfrak{X}_{\mu}(M)$ models passive scalar motion [17]:

$$
\begin{aligned}
\partial_{t} u & =-\nabla_{u} u-\operatorname{grad} p, \\
\partial_{t} f & =-d f(u) .
\end{aligned}
$$

In this case the $L^{2}$ scalar product on $C^{\infty}(M)$ is $\mathfrak{X}_{\mu}(M)$-invariant and we apply Corollary 1 to get this geodesic equation.

\section{Magnetohydrodynamics}

Let $A: \mathfrak{g} \rightarrow \mathfrak{g}^{*}$ be the inertia operator defined by a fixed scalar product $\langle$,$\rangle on \mathfrak{g}$. The scalar product on the regular dual $\mathfrak{g}_{\text {reg }}^{*}=A(\mathfrak{g})$ induced via $A$ by this scalar product in $\mathfrak{g}$ is again denoted by $\langle$,$\rangle . Next we consider the subgroup \mathfrak{g}_{\text {reg }}^{*} \rtimes G$ of the magnetic extension $\mathfrak{g}^{*} \rtimes G$, with right invariant metric of type (6.1) [56]. 
Proposition 2. If the adjoint of $\operatorname{ad}(X)$ exists for any $X \in \mathfrak{g}$, then the geodesic equation on the magnetic extension $\mathfrak{g}_{\mathrm{reg}}^{*} \rtimes G$ with right invariant metric, written for the curve $(A(v), u)$ in $\mathfrak{g}_{\mathrm{reg}}^{*} \rtimes \mathfrak{g}$ is

$$
\begin{aligned}
\frac{d}{d t} u & =-\operatorname{ad}(u)^{\top} u+\operatorname{ad}(v)^{\top} v, \\
\frac{d}{d t} v & =\operatorname{ad}(u) v .
\end{aligned}
$$

Proof. We have to compute the map $l: \mathfrak{g}_{\text {reg }}^{*} \times \mathfrak{g}_{\text {reg }}^{*} \rightarrow \mathfrak{g}$ and the adjoint $b(X)^{\top}: \mathfrak{g}_{\text {reg }}^{*} \rightarrow \mathfrak{g}_{\text {reg }}^{*}$ for $b=\mathrm{ad}^{*}$. We use the fact (2.3) that the coadjoint action on the image of $A$ comes from the opposite of $\operatorname{ad}(\cdot)^{\top}$. Then $l\left(A\left(Y_{1}\right), A\left(Y_{2}\right)\right)=\operatorname{ad}\left(Y_{2}\right)^{\top} Y_{1}$ because

$$
\left\langle l\left(A\left(Y_{1}\right), A\left(Y_{2}\right)\right), X\right\rangle=\left\langle\operatorname{ad}^{*}(X) A\left(Y_{1}\right), A\left(Y_{2}\right)\right\rangle=-\left\langle\operatorname{ad}(X)^{\top} Y_{1}, Y_{2}\right\rangle=\left\langle\operatorname{ad}\left(Y_{2}\right)^{\top} Y_{1}, X\right\rangle .
$$

Also the adjoint of $b(X)=\operatorname{ad}^{*}(X)$ exists and $b(X)^{\top} A(Y)=-A(\operatorname{ad}(X) Y)$. The result follows now from Corollary 1.

For $G=S O(3)$ and left invariant metric on its magnetic extension $\mathfrak{g}^{*} \rtimes G$ one obtains Kirchhoff equations for a rigid body moving in a fluid.

Let $G=\operatorname{Diff}_{\mu}(M)$ be the group of volume preserving diffeomorphisms on a compact manifold $M$ and $\mathfrak{g}=\mathfrak{X}_{\mu}(M)$. The regular part $\mathfrak{g}_{\text {reg }}^{*}$ of $\mathfrak{g}^{*}$ is naturally isomorphic to the quotient space $\Omega^{1}(M) / d \Omega^{0}(M)$ of differential 1-forms modulo exact 1-forms, the pairing being $([\alpha], X)=$ $\int_{M} \alpha(X) \mu$, for $\alpha \in \Omega^{1}(M)$. More precisely $A(X)$ is the coset $\left[X^{b}\right]$ obtained via the Riemannian metric.

Considering the right invariant $L^{2}$ metric on the magnetic extension $\mathfrak{g}_{\mathrm{reg}}^{*} \rtimes G$ determined by the $L^{2}$ scalar product (3.1) on vector fields, the geodesic equations for the time dependent divergence free vector fields $u$ and $B$ are (by Proposition 2)

$$
\begin{aligned}
& \partial_{t} u=-\nabla_{u} u+\nabla_{B} B-\operatorname{grad} p, \\
& \partial_{t} B=-L_{u} B .
\end{aligned}
$$

We specialize to a three dimensional manifold $M$. The curl of a vector field $X$ is the vector field defined by the relation $i_{\operatorname{curl} X} \mu=d X^{\mathrm{b}}$ and the cross product of two vector fields $X$ and $Y$ is the vector field defined by the relation $(X \times Y)^{b}=i_{Y} i_{X} \mu$. A short computation gives $(\operatorname{curl} X \times X)^{b}=i_{X} d X^{b}=L_{X} X^{b}-d g(X, X)=\left(\nabla_{X} X\right)^{b}-\frac{1}{2} d g(X, X)$, hence $\nabla_{X} X=\operatorname{curl} X \times$ $X+\frac{1}{2} \operatorname{grad} g(X, X)$. The geodesic equations above are in this case the equations of ideal magnetohydrodynamics with velocity $u$, magnetic field $B$ and pressure $p$ [54, 32]:

$$
\begin{aligned}
& \partial_{t} u=-\nabla_{u} u+\operatorname{curl} B \times B-\operatorname{grad} p, \\
& \partial_{t} B=-L_{u} B .
\end{aligned}
$$

\section{Magnetic hydrodynamics with asymmetric stress tensor}

Let $M$ be a 3-dimensional compact parallelizable Riemannian manifold with induced volume form $\mu$ and let $G=\operatorname{Diff}_{\mu}(M)$ with $\mathfrak{g}=\mathfrak{X}_{\mu}(M)$. Each vector field $X$ on $M$ can be identified with a smooth function in $C^{\infty}\left(M, \mathbb{R}^{3}\right)$, and $j(X) \in C^{\infty}(M, \mathfrak{g l}(3, \mathbb{R}))$ denotes its Jacobian. Then $\omega(X, Y)=[\operatorname{tr}(j(X) d j(Y))] \in \Omega^{1}(M) / d \Omega^{0}(M)$ is a Lie algebra 2-cocycle on $\mathfrak{g}$ with values in the regular dual $\mathfrak{g}_{\text {reg }}^{*}$.

Considering the $L^{2}$ scalar product on the Abelian extension $\mathfrak{g}_{\text {reg }}^{*} \rtimes_{\omega} \mathfrak{g}$, we get the following Euler equation [5] for time dependent divergence free vector fields $u$ and $B$ :

$$
\begin{aligned}
& \partial_{t} u=-\nabla_{u} u+\operatorname{curl} B \times B+\operatorname{tr}(j(B) \operatorname{grad} j(u))-\operatorname{grad} p, \\
& \partial_{t} B=-L_{u} B,
\end{aligned}
$$

modeling magnetic hydrodynamics with asymmetric stress tensor $T=j(B) \circ j(u)$. 


\section{Geodesic equations on central extensions}

When $V=\mathbb{R}$ is the trivial $\mathfrak{g}$-module, then the Lie algebra action $b$ vanishes and we get a central extension $\mathbb{R} \times_{\omega} \mathfrak{g}$ defined by the cocycle $\omega: \mathfrak{g} \times \mathfrak{g} \rightarrow \mathbb{R}$. A consequence of Proposition 1 is:

Corollary 2. The geodesic equation on a 1-dimensional central Lie group extension $\hat{G}$ of $G$ with right invariant metric determined by the scalar product $\langle(a, X),(b, Y)\rangle_{\hat{\mathfrak{g}}}=\langle X, Y\rangle_{\mathfrak{g}}+a b$ on its Lie algebra $\hat{\mathfrak{g}}=\mathbb{R} \times \omega \mathfrak{g}$ is

$$
\frac{d}{d t} u=-\operatorname{ad}(u)^{\top} u-a k(u), \quad a \in \mathbb{R},
$$

where $u$ is a curve in $\mathfrak{g}$ and $k \in L_{\text {skew }}(\mathfrak{g})$ is defined by the Lie algebra cocycle $\omega$ via

$$
\langle k(X), Y\rangle=\omega(X, Y), \quad \forall X, Y \in \mathfrak{g} .
$$

Proof. The central extension is a particular case of an Abelian extension, so Proposition 1 can be applied. The linear map $h: \mathbb{R} \rightarrow L_{\text {skew }}(\mathfrak{g})$ has the form $h(a) X=a k(X)$, because $\left\langle h(a) X_{1}, X_{2}\right\rangle_{\mathfrak{g}}=a \omega\left(X_{1}, X_{2}\right)=\left\langle a k\left(X_{1}\right), X_{2}\right\rangle_{\mathfrak{g}}$. The $\mathfrak{g}$-module $\mathbb{R}$ being trivial, $\frac{d}{d t} a=0$, so $a \in \mathbb{R}$ is constant.

\section{KdV equation}

The geodesic equation on the Bott-Virasoro group (5.2) for the right invariant $L^{2}$ metric is the Korteweg-de Vries equation [44]. In this case the Lie algebra is the central extension of $\mathfrak{g}=$ $\mathfrak{X}\left(S^{1}\right)$ (identified with $\left.C^{\infty}\left(S^{1}\right)\right)$ given by the Virasoro cocycle $\omega(X, Y)=\int_{S^{1}}\left(X^{\prime} Y^{\prime \prime}-X^{\prime \prime} Y^{\prime}\right) d x$. The computation $\omega(X, Y)=-2 \int_{S^{1}} X^{\prime \prime} Y^{\prime} d x=2 \int_{S^{1}} X^{\prime \prime \prime} Y d x=\left\langle X^{\prime \prime \prime}, Y\right\rangle$ implies $k(X)=2 X^{\prime \prime \prime}$ and by Corollary 2 the geodesic equation for $u: I \rightarrow C^{\infty}\left(S^{1}\right)$ is the $\mathrm{KdV}$ equation:

$$
\partial_{t} u=-3 u u^{\prime}-2 a u^{\prime \prime \prime}, \quad a \in \mathbb{R} .
$$

\section{Superconductivity equation}

Given a compact manifold $M$ with volume form $\mu$, each closed 2-form $\eta$ on $M$ defines a Lichnerowicz 2-cocycle $\omega_{\eta}$ on the Lie algebra of divergence free vector fields,

$$
\omega_{\eta}(X, Y)=\int_{M} \eta(X, Y) \mu
$$

The kernel of the flux homomorphism

$$
\operatorname{flux}_{\mu}: X \in \mathfrak{X}_{\mu}(M) \mapsto\left[i_{X} \mu\right] \in H^{n-1}(M, \mathbb{R})
$$

is the Lie algebra $\mathfrak{X}_{\mu}^{\mathrm{ex}}(M)$ of exact divergence free vector fields. On a 2-dimensional manifold it consists of vector fields $X$ possessing stream functions $f \in C^{\infty}(M)$, i.e. $i_{X} \mu=d f(X$ is the Hamiltonian vector field with Hamiltonian function $f$ ). On a 3-dimensional manifold it consists of vector fields $X$ possessing vector potentials $A \in \mathfrak{X}(M)$, i.e. $i_{X} \mu=d A^{b}$ ( $X$ is the curl of $A$ ).

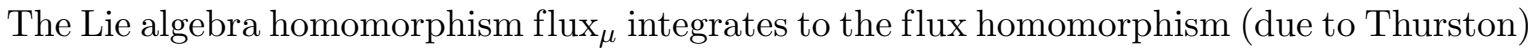
Flux $_{\mu}$ on the identity component of the group of volume preserving diffeomorphisms:

$$
\operatorname{Flux}_{\mu}: \operatorname{Diff}_{\mu}(M)_{0} \rightarrow H^{n-1}(M, \mathbb{R}) / \Gamma, \quad \operatorname{Flux}_{\mu}(\varphi)=\int_{0}^{1}\left[i_{\delta^{r} \varphi(t)} \mu\right] d t \quad \bmod \Gamma
$$


where $\varphi(t)$ is any volume preserving diffeotopy from the identity on $M$ to $\varphi$ and $\Gamma$ a discrete subgroup of $H^{n-1}(M, \mathbb{R})$. The kernel of Flux ${ }_{\mu}$ is, by definition, the Lie group $\operatorname{Diff}_{\mu}^{e x}(M)$ of exact volume preserving diffeomorphisms. It coincides with $\operatorname{Diff}_{\mu}(M)_{0}$ if and only if $H^{n-1}(M, \mathbb{R})=0$.

For $\eta$ integral, the Lichnerowicz cocycle is integrable to $\operatorname{Diff}_{\mu}^{\mathrm{ex}}(M)$ [23]. When $M$ is 3dimensional, there exists a vector field $B$ on $M$ defined with $\eta=-i_{B} \mu$. The 2 -form $\eta$ is closed if and only if $B$ is divergence free. The integrality condition of $\eta$ expresses as $\int_{S}(B \cdot n) d \sigma \in \mathbb{Z}$ on every closed surface $S \subset M$.

The superconductivity equation models the motion of a high density electronic gas in a magnetic field $B$ with velocity $u$ :

$$
\partial_{t} u=-\nabla_{u} u-a u \times B-\operatorname{grad} p, \quad a \in \mathbb{R} .
$$

It is the geodesic equation on a central extension of the group of volume preserving diffeomorphisms for the right invariant $L^{2}$ metric [61, 57], when $M$ is simply connected.

Indeed,

$$
\omega_{\eta}(X, Y)=\int_{M} \eta(X, Y) \mu=-\int_{M} \mu(B, X, Y) \mu=\int_{M} g(X \times B, Y) \mu=\langle P(X \times B), Y\rangle
$$

hence the map $k \in L_{\text {skew }}(\mathfrak{g})$ determined by the Lichnerowicz cocycle $\omega_{\eta}$ is $k(X)=P(X \times B)$, with $P$ denoting the orthogonal projection on the space of divergence free vector fields. Now we apply Corollary 2 .

\section{Charged ideal fluid}

Let $M$ be an $n$-dimensional Riemannian manifold with Levi-Civita connection $\nabla$ and volume form $\mu$, and $\eta$ a closed integral differential two-form. Let $B$ be an $(n-2)$ vector field on $M$ (i.e. $\left.B \in C^{\infty}\left(\wedge^{n-2} T M\right)\right)$ such that $\eta=(-1)^{n-2} i_{B} \mu$ is a closed two-form. The cross product of a vector field $X$ with $B$ is the vector field $X \times B=\left(i_{X \wedge B} \mu\right)^{\sharp}=\left(i_{X} \eta\right)^{\sharp}, \sharp$ denoting the Riemannian lift. When $M$ is 3-dimensional, then $B$ is a divergence free vector field with $\eta=-i_{B} \mu$ and $\times$ is the cross product of vector fields.

From the integrality of $\eta$ follows the existence of a principal $\mathbb{T}$-bundle $\pi: P \rightarrow M$ with a principal connection 1-form $\alpha$ on $P$ having curvature $\eta$. The associated Kaluza-Klein metric $\kappa$ on $P$, defined at a point $x \in P$ by

$$
\kappa_{x}(\tilde{X}, \tilde{Y})=g_{\pi(x)}\left(T_{x} \pi \cdot \tilde{X}, T_{x} \pi \cdot \tilde{Y}\right)+\alpha_{x}(\tilde{X}) \alpha_{x}(\tilde{Y}), \quad \tilde{X}, \tilde{Y} \in T_{x} P
$$

determines the volume form $\tilde{\mu}=\pi^{*} \mu \wedge \alpha$ on $P$.

The group $\operatorname{Diff}_{\tilde{\mu}}(P)^{\mathbb{T}}$ of volume preserving automorphisms of the principal bundle $P$ is an Abelian Lie group extension of $\operatorname{Diff}_{\mu}(M)_{[P]}$, the group of volume preserving diffeomorphisms preserving the bundle class $[P]$, by the gauge group $C^{\infty}(M, \mathbb{T})$ (an extension contained in (5.3)). The corresponding Abelian Lie algebra extension

$$
0 \rightarrow C^{\infty}(M) \rightarrow \mathfrak{X}_{\tilde{\mu}}(P)^{\mathbb{T}} \rightarrow \mathfrak{X}_{\mu}(M) \rightarrow 0
$$

is described again by the Lie algebra cocycle $\omega: \mathfrak{X}_{\mu}(M) \times \mathfrak{X}_{\mu}(M) \rightarrow C^{\infty}(M)$ given by $\eta$.

The Kaluza-Klein metric on $P$ determines a right invariant $L^{2}$ metric on the group of volume preserving automorphisms of the principal $\mathbb{T}$-bundle $P$. The geodesic equation written in terms of the right logarithmic derivative $(\rho, u)$, with $\rho$ a time dependent function and $u$ a time dependent divergence free vector field on $M$, is:

$$
\begin{aligned}
& \partial_{t} u=-\nabla_{u} u-\rho u \times B-\operatorname{grad} p, \\
& \partial_{t} \rho=-d \rho(u) .
\end{aligned}
$$


It models the motion of a charged ideal fluid with velocity $u$, pressure $p$ and charge density $\rho$ in a fixed magnetic field $B$ [57].

Indeed, the connection $\alpha$ defines a horizontal lift and identifying the pair $(f, X), f \in C^{\infty}(M)$, $X \in \mathfrak{X}_{\mu}(M)$ with the sum of the horizontal lift of $X$ and the vertical vector field given by $f$, we get an isomorphism between the Abelian Lie algebra extension $C^{\infty}(M) \rtimes_{\omega} \mathfrak{X}_{\mu}(M)$ and the Lie algebra $\mathfrak{X}_{\tilde{\mu}}(P)^{\mathbb{T}}$ of invariant divergence free vector fields on $P$. Under this isomorphism the $L^{2}$ metric defined by the Kaluza-Klein metric $\kappa$ is $\left\langle\left(f_{1}, X_{1}\right),\left(f_{2}, X_{2}\right)\right\rangle=\int_{M}\left(g\left(X_{1}, X_{2}\right)+f_{1} f_{2}\right) \mu$. The $L^{2}$ scalar product on functions is $\mathfrak{X}_{\mu}(M)$ invariant, i.e. $b(X)$ is skew-adjoint. The mapping $h: C^{\infty}(M) \rightarrow L_{\text {skew }}\left(\mathfrak{X}_{\mu}(M)\right)$ is $h(f) X=P(f X \times B)$ because:

$$
\langle h(f) X, Y\rangle=\langle\eta(X, Y), f\rangle=\int_{M} f\left(i_{X} \eta\right)(Y) \mu=\int_{M} f g(X \times B, Y) \mu=\langle P(f X \times B), Y\rangle,
$$

where $P$ denotes the orthogonal projection on the space of divergence free vector fields on $M$. The result follows from Remark 3, knowing that $\operatorname{ad}(X)^{\top} X=P\left(\nabla_{X} X\right)$.

\section{Geodesics on general extensions}

A general extension of Lie algebras is an exact sequence of Lie algebras

$$
0 \rightarrow \mathfrak{h} \rightarrow \hat{\mathfrak{g}} \rightarrow \mathfrak{g} \rightarrow 0
$$

A section $s: \mathfrak{g} \rightarrow \hat{\mathfrak{g}}$ (i.e. a right inverse to the projection $\hat{\mathfrak{g}} \rightarrow \mathfrak{g}$ ) induces the following mappings [1]:

$$
\begin{array}{ll}
b: & \mathfrak{g} \rightarrow \operatorname{Der}(\mathfrak{h}), \quad b(X) f=[s(X), f], \\
\omega: & \mathfrak{g} \times \mathfrak{g} \rightarrow \mathfrak{h}, \quad \omega\left(X_{1}, X_{2}\right)=\left[s\left(X_{1}\right), s\left(X_{2}\right)\right]-s\left(\left[X_{1}, X_{2}\right]\right)
\end{array}
$$

with properties:

$$
\begin{aligned}
& {\left[b\left(X_{1}\right), b\left(X_{2}\right)\right]-b\left(\left[X_{1}, X_{2}\right]\right)=\operatorname{ad}\left(\omega\left(X_{1}, X_{2}\right)\right),} \\
& \sum_{\text {cycl }} \omega\left(\left[X_{1}, X_{2}\right], X_{3}\right)=\sum_{\text {cycl }} b\left(X_{1}\right) \omega\left(X_{2}, X_{3}\right) .
\end{aligned}
$$

The Lie algebra structure on the extension $\hat{\mathfrak{g}}$, identified as a vector space with $\mathfrak{h} \oplus \mathfrak{g}$ via the section $s$, can be expressed in terms of $b$ and $\omega$ :

$$
\left[\left(f_{1}, X_{1}\right),\left(f_{2}, X_{2}\right)\right]=\left(\left[f_{1}, f_{2}\right]+b\left(X_{1}\right) f_{2}-b\left(X_{2}\right) f_{1}+\omega\left(X_{1}, X_{2}\right),\left[X_{1}, X_{2}\right]\right) .
$$

In particular for $\mathfrak{h}$ an Abelian Lie algebra this is the Lie bracket (5.1) on an Abelian Lie algebra extension.

We consider scalar products $\langle,\rangle_{\mathfrak{g}}$ on $\mathfrak{g}$ and $\langle,\rangle_{\mathfrak{h}}$ on $\mathfrak{h}$ and, as in Section 6 , we impose the existence of several maps: $\operatorname{ad}(X)^{\top}: \mathfrak{g} \rightarrow \mathfrak{g}$ for any $X \in \mathfrak{g}, \operatorname{ad}(f)^{\top}: \mathfrak{h} \rightarrow \mathfrak{h}$ for any $f \in \mathfrak{h}$, $b(X)^{\top}: \mathfrak{h} \rightarrow \mathfrak{h}$ for any $X \in \mathfrak{g}$, as well as the linear map $h: \mathfrak{h} \rightarrow L_{\text {skew }}(\mathfrak{g})$ defined by

$$
\left\langle h(f) X_{1}, X_{2}\right\rangle_{\mathfrak{g}}=\left\langle\omega\left(X_{1}, X_{2}\right), f\right\rangle_{\mathfrak{h}},
$$

and the bilinear map $l: \mathfrak{h} \times \mathfrak{h} \rightarrow \mathfrak{g}$, defined by

$$
\left\langle l\left(f_{1}, f_{2}\right), X\right\rangle_{\mathfrak{g}}=\left\langle b(X) f_{1}, f_{2}\right\rangle_{\mathfrak{h}} .
$$

A result similar to Proposition 1 is: 
Proposition 3. The geodesic equation on the Lie group extension $\hat{G}$ of $G$ by $H$ integrating (12.1), with right invariant metric determined by the scalar product

$$
\left\langle\left(f_{1}, X_{1}\right),\left(f_{2}, X_{2}\right)\right\rangle_{\hat{\mathfrak{g}}}=\left\langle f_{1}, f_{2}\right\rangle_{\mathfrak{h}}+\left\langle X_{1}, X_{2}\right\rangle_{\mathfrak{g}}
$$

written in terms of the right logarithmic derivative $(\rho, u)$ is:

$$
\begin{aligned}
& \frac{d}{d t} u=-\operatorname{ad}(u)^{\top} u-h(\rho) u+l(\rho, \rho), \\
& \frac{d}{d t} \rho=-\operatorname{ad}(\rho)^{\top} \rho-b(u)^{\top} \rho .
\end{aligned}
$$

\section{Ideal fluid in a fixed Yang-Mills field}

Let $\pi: P \rightarrow M$ be a principal $G$-bundle with principal action $\sigma: P \times G \rightarrow P$ and let $\operatorname{Ad} P=$ $P \times_{G} \mathfrak{g}$ be its adjoint bundle. The space $\Omega^{k}(M, \operatorname{Ad} P)$ of differential forms with values in $\operatorname{Ad} P$ is identified with the space $\Omega_{\mathrm{hor}}^{k}(P, \mathfrak{g})^{G}$ of $G$-equivariant horizontal forms on $P$. In particular $C^{\infty}(M, \operatorname{Ad} P)=C^{\infty}(P, \mathfrak{g})^{G}$.

We consider a principal connection 1-form $\alpha \in \Omega^{1}(P, \mathfrak{g})^{G}$ on $P$. Its curvature $\eta=d \alpha+\frac{1}{2}[\alpha, \alpha]$. is an equivariant horizontal 2 -form $\eta \in \Omega_{\mathrm{hor}}^{2}(P, \mathfrak{g})^{G}$, hence it can be viewed as a 2 -form on $M$ with values in $\operatorname{Ad} P$. The covariant exterior derivative on $\mathfrak{g}$-valued differential forms on $P$ is $d^{\alpha}=\chi^{*} \circ d$, with $\chi: \mathfrak{X}(P) \rightarrow \mathfrak{X}(P)$ denoting the horizontal projection, and it induces a map $d^{\alpha}: \Omega^{k}(M, \operatorname{Ad} P) \rightarrow \Omega^{k+1}(M, \operatorname{Ad} P)$.

Let $g$ be a Riemannian metric on $M$ and $\gamma$ a $G$-invariant scalar product on $\mathfrak{g}$. These data, together with the connection $\alpha$, define a Kaluza-Klein metric on $P$ :

$$
\kappa_{x}(\tilde{X}, \tilde{Y})=g_{\pi(x)}\left(T_{x} \pi \cdot \tilde{X}, T_{x} \pi . \tilde{Y}\right)+\gamma\left(\alpha_{x}(\tilde{X}), \alpha_{x}(\tilde{Y})\right), \quad \tilde{X}, \tilde{Y} \in T_{x} P .
$$

The canonically induced volume form on $P$ is $\tilde{\mu}=\pi^{*} \mu \wedge \alpha^{*} \operatorname{det}_{\gamma}$, where $\mu$ is the canonical volume form on $M$ induced by the Riemannian metric $g$ and $\alpha^{*} \operatorname{det}_{\gamma}$ is the pullback by $\alpha: T P \rightarrow \mathfrak{g}$ of the determinant $\operatorname{det}_{\gamma} \in \wedge^{\operatorname{dim}} \mathfrak{g}_{\mathfrak{g}}$ induced by the scalar product $\gamma$ on $\mathfrak{g}$.

The gauge group of the principal bundle is identified with $C^{\infty}(P, G)^{G}$, the group of $G$ equivariant functions from $P$ to $G$, with $G$ acting on itself by conjugation. The group of automorphisms of $P$, i.e. the group of $G$-equivariant diffeomorphisms of $P$, is an extension of $\operatorname{Diff}(M)_{[P]}$, the group of diffeomorphisms of $M$ preserving the bundle class $[P]$, by the gauge group. This is the analogue of (5.3) for non-commutative structure group. Restricting to volume preserving diffeomorphisms, we get the exact sequence:

$$
1 \rightarrow C^{\infty}(P, G)^{G} \stackrel{\sigma}{\rightarrow} \operatorname{Diff}_{\tilde{\mu}}(P)^{G} \rightarrow \operatorname{Diff}_{\mu}(M)_{[P]} \rightarrow 1 .
$$

On the Lie algebra level the exact sequence is

$$
0 \rightarrow C^{\infty}(P, \mathfrak{g})^{G} \stackrel{\dot{\sigma}}{\rightarrow} \mathfrak{X}_{\tilde{\mu}}(P)^{G} \rightarrow \mathfrak{X}_{\mu}(M) \rightarrow 0 .
$$

The horizontal lift provides a linear section : $\mathfrak{X}_{\mu}(M) \rightarrow \mathfrak{X}_{\tilde{\mu}}(P)^{G}$, thus identifying the pair $(f, X) \in C^{\infty}(P, \mathfrak{g})^{G} \oplus \mathfrak{X}_{\mu}(M)$ with $\tilde{X}=\dot{\sigma}(f)+X^{\text {hor }} \in \mathfrak{X}_{\tilde{\mu}}(P)^{G}$. With this identification, the $L^{2}$ metric on $\mathfrak{X}_{\tilde{\mu}}(P)^{G}$ given by the Kaluza-Klein metric can be written as

$$
\left.\int_{P} \kappa\left(f_{1}, X_{1}\right),\left(f_{2}, X_{2}\right)\right) \tilde{\mu}=\int_{M} g\left(X_{1}, X_{2}\right) \mu+\int_{P} \gamma\left(f_{1}, f_{2}\right) \tilde{\mu} .
$$

A particular case of a result in [14] is the fact that the geodesic equation on the group $\operatorname{Diff}_{\tilde{\mu}}(P)^{G}$ of volume preserving automorphisms of $P$ with right invariant $L^{2}$ metric gives the 
equations of motion of an ideal fluid moving in a fixed Yang-Mills field. Written for the right logarithmic derivative $(\rho, u): I \rightarrow C^{\infty}(P, \mathfrak{g})^{G} \oplus \mathfrak{X}_{\mu}(M)$, these are:

$$
\begin{aligned}
& \partial_{t} u=-\nabla_{u} u-\gamma\left(\rho, i_{u} \eta\right)^{\sharp}-\operatorname{grad} p, \\
& \partial_{t} \rho=-d^{\alpha} \rho(u) .
\end{aligned}
$$

Here $u$ denotes the Eulerian velocity, $\rho$, viewed as a time dependent section of $\operatorname{Ad} P$, denotes the magnetic charge, $\eta$, viewed as a 2 -form on $M$ with values in Ad $P$, denotes the fixed Yang-Mills field. The scalar product $\gamma$ being $G$-invariant, can be viewed as a bundle metric on $\operatorname{Ad} P$.

This result follows from Proposition 3. Indeed, in this particular case the cocycle is $\omega=\eta$ and the Lie algebra action is $b(X) f=-d f \cdot X^{\text {hor }}=-d^{\alpha} f \cdot X$, hence $b(X)^{\top} f=-b(X) f, l$ is skewsymmetric and $h(f) X=P\left(\gamma\left(f, i_{X} \eta\right)^{\sharp}\right)$. Moreover $\operatorname{ad}(X)^{\top} X=P \nabla_{X} X$ with $P$ the projection on divergence free vector fields and $\operatorname{ad}(f)^{\top} f=[f, f]=0$, so (13.1) follows.

The equations of a charged ideal fluid from Section 11 are obtained for the structure group $G$ equal to the torus $\mathbb{T}$.

\section{Totally geodesic subgroups}

Let $G$ be a Lie group with right invariant Riemannian metric. A Lie subgroup $H \subseteq G$ is totally geodesic if any geodesic $c:[a, b] \rightarrow G$ with $c(a)=e$ and $c^{\prime}(a) \in \mathfrak{h}$, the Lie algebra of $H$, stays in $H$.

From the Euler equation (2.1) we see that this is the case if $\operatorname{ad}(X)^{\top} X \in \mathfrak{h}$ for all $X \in \mathfrak{h}$. If there is a geodesic in $G$ in any direction of $\mathfrak{h}$, then this condition is necessary and sufficient, so we give the following definition: the Lie subalgebra $\mathfrak{h}$ is called totally geodesic in $\mathfrak{g}$ if $\operatorname{ad}(X)^{\top} X \in \mathfrak{h}$ for all $X \in \mathfrak{h}$.

Remark 4. Given two totally geodesic Lie subalgebras $\mathfrak{h}$ and $\mathfrak{k}$ of the Lie algebra $\mathfrak{g}$, the intersection $\mathfrak{h} \cap \mathfrak{k}$ is totally geodesic in $\mathfrak{g}$, but also in $\mathfrak{h}$ and in $\mathfrak{k}$.

\section{Ideal fluid}

The ideal fluid flow (3.2) on $M$ preserves the property of having a stream function (if $M$ two dimensional), resp. a vector potential (if $M$ three dimensional) if and only if $\operatorname{Diff}_{\mu}^{\mathrm{ex}}(M)$ is a totally geodesic subgroup of $\operatorname{Diff}_{\mu}(M)$ for the right invariant $L^{2}$ metric. This means $P\left(\nabla_{X} X\right) \in \mathfrak{X}_{\mu}^{\text {ex }}(M)$ for all $X \in \mathfrak{X}_{\mu}^{\mathrm{ex}}(M)$.

Theorem 2 ([16]). The only Riemannian manifolds $M$ with the property that $\operatorname{Diff}_{\mu}^{\mathrm{ex}}(M)$ is a totally geodesic subgroup of $\operatorname{Diff}_{\mu}(M)$ with the right invariant $L^{2}$ metric are twisted products $M=\mathbb{R}^{k} \times{ }_{\Lambda} F$ of a flat torus $\mathbb{T}^{k}=\mathbb{R}^{k} / \Lambda$ and a connected oriented Riemannian manifold $F$ with $H^{1}(F, \mathbb{R})=0$.

In particular the ideal fluid flow on the 2-torus preserves the property of having a stream function [3] and the ideal fluid flow on the 3-torus preserves the property of having a vector potential.

\section{Superconductivity}

Given a compact Riemannian manifold $M$, from the Hodge decomposition follows that $\mathfrak{X}_{\mu}(M)=$ $\mathfrak{X}_{\mu}^{\mathrm{ex}}(M) \oplus \mathfrak{X}_{\text {harm }}(M)$. On a flat torus the harmonic vector fields are those with all components constant.

In the setting of Section 10, the next proposition determines when is $\mathbb{R} \rtimes_{\omega_{\eta}} \mathfrak{X}_{\mu}^{\operatorname{ex}}(M)$ totally geodesic in $\mathbb{R} \rtimes_{\omega_{\eta}} \mathfrak{X}_{\mu}(M)$, for $M=\mathbb{T}^{3}$ and $\eta=-i_{B} \mu$. 
Proposition 4 ([58]). The superconductivity equation (10.1) on the 3 -torus preserves the property of having a vector potential if and only if the three components of the magnetic field $B$ are constant.

Proof. Any exact divergence free vector field $X$ on the 3 -torus admits a potential 1-form $\alpha$ with $i_{X} \mu=d \alpha$, hence $\int_{\mathbb{T}^{3}} g(X \times B, Y) \mu=\int_{\mathbb{T}^{3}} i_{Y} i_{B} \mu \wedge i_{X} \mu=\int_{\mathbb{T}^{3}} i_{[Y, B]} \mu \wedge \alpha$. Then the totally geodesicity condition which, in this case, says that $P(X \times B)$ is exact divergence free for all $X$ exact divergence free, is equivalent to $[Y, B]=0$ for all harmonic vector fields $Y$. This is further equivalent to the fact that the three components of the magnetic field $B$ are constant.

\section{Passive scalar motion}

On the trivial principal $\mathbb{T}$ bundle $P=M \times \mathbb{T}$ we consider the volume form $\tilde{\mu}=\mu \wedge d \theta$. Noticing that $i_{(f, X)} \tilde{\mu}=i_{X} \mu \wedge d \theta+f \mu$, we get the Lie algebra isomorphisms $\mathfrak{X}_{\tilde{\mu}}(M \times \mathbb{T})^{\mathbb{T}} \cong C^{\infty}(M) \rtimes \mathfrak{X}_{\mu}(M)$ and $\mathfrak{X}_{\tilde{\mu}}^{\mathrm{ex}}(M \times \mathbb{T})^{\mathbb{T}} \cong C_{0}^{\infty}(M) \rtimes \mathfrak{X}_{\mu}^{\mathrm{ex}}(M)$, where $C_{0}^{\infty}(M)$ is the subspace of functions with vanishing integral.

From [55] we know that the group of equivariant volume preserving diffeomorphisms is totally geodesic in the group of volume preserving diffeomorphisms and from Theorem 2 we know that the group of exact volume preserving diffeomorphisms of a torus is totally geodesic in the group of volume preserving diffeomorphisms, hence by Remark 4 we obtain that for $M=\mathbb{T}^{2}$ the subgroup $\operatorname{Diff}_{\tilde{\mu}}^{\operatorname{ex}}(M \times \mathbb{T})^{\mathbb{T}}$ is totally geodesic in $\operatorname{Diff}_{\tilde{\mu}}(M \times \mathbb{T})^{\mathbb{T}}$. This means that $C_{0}^{\infty}(M) \rtimes \mathfrak{X}_{\mu}^{\mathrm{ex}}(M)$ is totally geodesic in $C^{\infty}(M) \rtimes \mathfrak{X}_{\mu}(M)$ for $M=\mathbb{T}^{2}$. In other words equation (7.1), describing passive scalar motion, preserves the property of having a stream function if $f$ has zero integral at the initial moment. Moreover, $f$ will have zero integral at any moment.

\section{Quasigeostrophic motion}

Given a closed 1-form $\alpha$ on the compact symplectic manifold $(M, \sigma)$, the Roger cocycle on the Lie algebra $\mathfrak{X}_{\sigma}^{\mathrm{ex}}(M)$ of Hamiltonian vector fields on $M$ is [49]

$$
\omega_{\alpha}\left(H_{f}, H_{g}\right)=\int_{M} f \alpha\left(H_{g}\right) \sigma^{n} .
$$

Here $f$ and $g$ are Hamiltonian functions with zero integral for the Hamiltonian vector fields $H_{f}$ and $H_{g}$. The integrability of the 2-cocycle $\omega_{\alpha}$ to a central extension of the group of Hamiltonian diffeomorphisms is an open problem. Partial results are given in [24].

For $M=\mathbb{T}^{2}$ the cocycle $\omega_{\alpha}$ can be extended to a cocycle on the Lie algebra of symplectic vector fields $\mathfrak{X}_{\sigma}\left(\mathbb{T}^{2}\right)$ by $\omega_{\alpha}\left(\partial_{x}, \partial_{y}\right)=\omega_{\alpha}\left(\partial_{x}, H_{f}\right)=\omega_{\alpha}\left(\partial_{y}, H_{f}\right)=0$ [27]. The extendability of $\omega_{\alpha}$ to $\mathfrak{X}_{\sigma}(M)$ for $M$ an arbitrary symplectic manifold is studied in [59]. To a divergence free vector field $X$ on the 2-torus one can assign a smooth function $\psi_{X}$ on the 2-torus uniquely determined by $X$ through $d \psi_{X}=i_{X} \sigma-\left\langle i_{X} \sigma\right\rangle$ and $\int_{\mathbb{T}^{2}} \psi_{X} \sigma=0$. Here \langle\rangle denotes the average of a 1-form

on the torus: $\langle a d x+b d y\rangle=\left(\int_{\mathbb{T}^{2}} a \sigma\right) d x+\left(\int_{\mathbb{T}^{2}} b \sigma\right) d y$. In particular $\psi_{H_{f}}=f$ whenever $f$ has zero integral.

Proposition 5 ([60]). The Euler equation for the $L^{2}$ scalar product on $\mathbb{R} \times_{\omega_{\alpha}} \mathfrak{X}_{\sigma}\left(\mathbb{T}^{2}\right)$ is

$$
\partial_{t} u=-\nabla_{u} u-\psi_{u} \alpha^{\sharp}-\operatorname{grad} p,
$$

where the function $\psi_{u}$ is uniquely determined by $u$ through $d \psi_{u}=i_{u} \sigma-\left\langle i_{u} \sigma\right\rangle$ and $\int_{\mathbb{T}^{2}} \psi_{u} \sigma=0$.

Proof. To apply Corollary 2 we compute the map $k$ corresponding to the cocycle $\omega_{\alpha}$. Using the fact that $\omega_{\alpha}\left(\partial_{x}, X\right)=\omega_{\alpha}\left(\partial_{y}, X\right)=0$ for all $X \in \mathfrak{X}_{\sigma}\left(\mathbb{T}^{2}\right)$, we get

$$
\omega_{\alpha}(u, X)=\omega_{\alpha}\left(H_{\psi_{u}}, X\right)=\int_{\mathbb{T}^{2}} \psi_{u} \alpha(X) \sigma=\int_{\mathbb{T}^{2}} g\left(\psi_{u} \alpha^{\sharp}, X\right) \sigma=\left\langle P\left(\psi_{u} \alpha^{\sharp}\right), X\right\rangle,
$$


hence $k(u)=P\left(\psi_{u} \alpha^{\sharp}\right)$. Knowing also that $\operatorname{ad}(u)^{\top} u=P\left(\nabla_{u} u\right)$, we get (15.1) as the Euler equation for $a=1$.

Proposition 6 ([60]). If the two components of the 1 -form $\alpha$ on $\mathbb{T}^{2}$ are constant, then equation (15.1) preserves the property of having a stream function, i.e. $\mathbb{R} \times_{\omega_{\alpha}} \mathfrak{X}_{\sigma}^{\mathrm{ex}}\left(\mathbb{T}^{2}\right)$ is totally geodesic in $\mathbb{R} \times_{\omega_{\alpha}} \mathfrak{X}_{\sigma}\left(\mathbb{T}^{2}\right)$. In this case the restriction of (15.1) to Hamiltonian vector fields is

$$
\partial_{t} H_{\psi}=-\nabla_{H_{\psi}} H_{\psi}-\psi \alpha^{\sharp}-\operatorname{grad} p .
$$

Proof. By Theorem 2 on the 2-torus $P\left(\nabla_{X} X\right)$ is Hamiltonian for $X$ Hamiltonian, hence the totally geodesicity condition in this case is equivalent to the fact that $P\left(\psi_{X} \alpha^{\sharp}\right)$ is Hamiltonian for $X$ Hamiltonian. By Hodge decomposition this means $\psi_{X} \alpha^{\sharp}$ is orthogonal to the space of harmonic vector fields, so

$$
\left\langle P\left(\psi_{X} \alpha^{\sharp}\right), Y\right\rangle=\int_{\mathbb{T}^{2}} g\left(\psi_{X} \alpha^{\sharp}, Y\right) \sigma=\int_{\mathbb{T}^{2}} \alpha(Y) \psi_{X} \sigma=0, \quad \forall Y \text { harmonic. }
$$

On the torus the harmonic vector fields $Y$ are the vector fields with constant components and the functions $\psi_{X}$ have vanishing integral by definition, so the expression above vanishes for all constant vector fields $Y$ if the 1-form $\alpha$ has constant coefficients.

On the 2-torus with $\sigma=d x \wedge d y$ and $u=H_{\psi}$, the vorticity 2-form is $d u^{b}=d\left(H_{\psi}\right)^{b}=(\Delta \psi) \sigma$, hence $\omega=\Delta \psi$ is the vorticity function. Since $L_{u}\left(d u^{b}\right)=L_{H_{\psi}}(\omega \sigma)=\left(L_{H_{\psi}} \omega\right) \sigma=\{\omega, \psi\} \sigma$, the vorticity equation (3.3) written for the vorticity function $\omega$ becomes

$$
\partial_{t} \omega=-\{\omega, \psi\}
$$

For $\alpha=\beta d y, \beta \in \mathbb{R}$, we have $d\left(\psi \alpha^{\sharp}\right)^{b}=d \psi \wedge \alpha=\left(\beta \partial_{x} \psi\right) \sigma$. Hence the Euler equation (15.2) written for the vorticity function $\omega=\Delta \psi$ with $\psi$ the stream function of $u$, is the equation for quasigeostrophic motion in $\beta$-plane approximation $[62,21]$

$$
\partial_{t} \omega=-\{\omega, \psi\}-\beta \partial_{x} \psi,
$$

with $\beta$ the gradient of the Coriolis parameter.

\section{Central extensions of semidirect products}

Let $\mathfrak{g}$ be a Lie algebra with scalar product $\langle,\rangle_{\mathfrak{g}}$ and $V$ a $\mathfrak{g}$-module with $\mathfrak{g}$-action $b$ and $\mathfrak{g}$-invariant scalar product $\langle,\rangle_{V}$. Each Lie algebra 1-cocycle $\alpha \in Z^{1}(\mathfrak{g}, V)$ (i.e. a linear map $\alpha: \mathfrak{g} \rightarrow V$ which satisfies $\left.\alpha\left(\left[X_{1}, X_{2}\right]\right)=b\left(X_{1}\right) \alpha\left(X_{2}\right)-b\left(X_{2}\right) \alpha\left(X_{1}\right)\right)$ defines a 2-cocycle $\omega$ on the semidirect product $V \rtimes \mathfrak{g}[45]$ :

$$
\omega\left(\left(v_{1}, X_{1}\right),\left(v_{2}, X_{2}\right)\right)=\left\langle\alpha\left(X_{1}\right), v_{2}\right\rangle_{V}-\left\langle\alpha\left(X_{2}\right), v_{1}\right\rangle_{V}
$$

Proposition 7. The Euler equation on the central extension $(\mathfrak{g} \ltimes V) \times_{\omega} \mathbb{R}$ with respect to the scalar product $\langle,\rangle_{\mathfrak{g}}+\langle,\rangle_{V}$, written for curves $u$ in $\mathfrak{g}$ and $f$ in $V$, is

$$
\begin{aligned}
& \frac{d}{d t} u=-\operatorname{ad}(u)^{\top} u+a \alpha^{\top}(f), \\
& \frac{d}{d t} f=b(u) f-a \alpha(u), \quad a \in \mathbb{R},
\end{aligned}
$$

where $\alpha^{\top}: V \rightarrow \mathfrak{g}$ is the adjoint of $\alpha: \mathfrak{g} \rightarrow V$. 
Proof. The map $k \in L_{\text {skew }}(V \rtimes \mathfrak{g})$ defined by $\omega$ is $k(v, X)=\left(\alpha(X),-\alpha^{\top}(v)\right)$ because

$$
\omega\left(\left(v_{1}, X_{1}\right),\left(v_{2}, X_{2}\right)\right)=\left\langle\alpha\left(X_{1}\right), v_{2}\right\rangle_{V}-\left\langle\alpha^{\top}\left(v_{1}\right), X_{2}\right\rangle_{\mathfrak{g}}=\left\langle\left(\alpha\left(X_{1}\right),-\alpha^{\top}\left(v_{1}\right)\right),\left(v_{2}, X_{2}\right)\right\rangle_{V \rtimes \mathfrak{g} .}
$$

The result follows from Corollaries 1 and 2 .

Remark 5. More generally, a 1-cocycle $\alpha$ on $\mathfrak{g}$ with values in the dual $\mathfrak{g}$-module $V^{*}$ defines a 2-cocycle on $V \rtimes \mathfrak{g}$ by

$$
\omega\left(\left(v_{1}, X_{1}\right),\left(v_{2}, X_{2}\right)\right)=\left(\alpha\left(X_{1}\right), v_{2}\right)-\left(\alpha\left(X_{2}\right), v_{1}\right),
$$

where $($,$) denotes the pairing between V^{*}$ and $V$.

\section{Stratified fluid}

Let $M$ be a compact Riemannian manifold with induced volume form $\mu$. Let $\alpha$ be a closed 1 -form on $M$. Then $\alpha: \mathfrak{X}(M) \rightarrow C^{\infty}(M)$ is a Lie algebra 1-cocycle with values in the canonical $\mathfrak{X}(M)$ module $C^{\infty}(M)$. The $L^{2}$ scalar product is $\mathfrak{X}_{\mu}(M)$-invariant, so $\alpha$ defines by (16.1) a 2-cocycle $\omega$ on the semidirect product Lie algebra $C^{\infty}(M) \rtimes \mathfrak{X}_{\mu}(M)$ :

$$
\omega\left(\left(f_{1}, X_{1}\right),\left(f_{2}, X_{2}\right)\right)=\int_{M} f_{2} \alpha\left(X_{1}\right) \mu-\int_{M} f_{1} \alpha\left(X_{2}\right) \mu .
$$

Proposition 8. The Euler equation on $\left(C^{\infty}(M) \rtimes \mathfrak{X}_{\mu}(M)\right) \times_{\omega} \mathbb{R}$ with $L^{2}$ scalar product is

$$
\begin{aligned}
& \partial_{t} u=-\nabla_{u} u+a f \alpha^{\sharp}-\operatorname{grad} p, \\
& \partial_{t} f=-L_{u} f-a \alpha(u), \quad a \in \mathbb{R},
\end{aligned}
$$

with $\nabla$ the Levi-Civita covariant derivative and $\sharp$ the Riemannian lift.

Proof. We apply Proposition 7 for $\mathfrak{g}=\mathfrak{X}_{\mu}(M)$ and $V=C^{\infty}(M)$. In this case $b(X) f=$ $-L_{X} f$ and $\operatorname{ad}(X)^{\top} X=P \nabla_{X} X$. We compute $\left\langle\alpha^{\top}(f), X\right\rangle_{\mathfrak{g}}=\int_{M} f \alpha(X) \mu=\int_{M} g\left(f \alpha^{\sharp}, X\right) \mu=$ $\left\langle P\left(f \alpha^{\sharp}\right), X\right\rangle_{\mathfrak{g}}$, for all $X \in \mathfrak{X}_{\mu}(M)$, hence $\alpha^{\top}(f)=P\left(f \alpha^{\sharp}\right)$.

Because $d\left(f \alpha^{\sharp}\right)^{b}=d f \wedge \alpha$, the equation (17.2) written for vorticity 2-form $\omega=d u^{b}$ becomes

$$
\begin{aligned}
& \partial_{t} \omega=-L_{u} \omega+d f \wedge \alpha, \\
& \partial_{t} f=-L_{u} f-a \alpha(u) .
\end{aligned}
$$

Proposition 9 ([58]). Given a 2-cocycle $\omega$ determined via (17.1) by the constant 1 -form $\alpha$ on the torus $M=\mathbb{T}^{2}$, we have $\left(\mathfrak{X}_{\mu}^{\mathrm{ex}}(M) \ltimes C_{0}^{\infty}(M)\right) \times{ }_{\omega} \mathbb{R}$ is totally geodesic in $\left(\mathfrak{X}_{\mu}(M) \ltimes C^{\infty}(M)\right) \times{ }_{\omega} \mathbb{R}$, where $C_{0}^{\infty}(M)$ is the subspace of functions with vanishing integral.

Proof. We know from Section 14 that for $M=\mathbb{T}^{2}, \mathfrak{X}_{\mu}^{\mathrm{ex}}(M) \ltimes C_{0}^{\infty}(M)$ is totally geodesic in $\mathfrak{X}_{\mu}(M) \ltimes C^{\infty}(M)$. But $\alpha(u)$ has zero integral for $u$ exact divergence free, $\alpha$ being closed. We have to make sure that $f \alpha^{\sharp}$ is orthogonal to the space of harmonic vector fields for all $f$ with zero integral, i.e. for all functions $f$ such that $f \mu$ is exact $(f \mu=d \nu)$. But $\int_{M} g\left(f \alpha^{\sharp}, Y\right) \mu=$ $\int_{M} \alpha(Y) d \nu=-\int_{M} L_{Y} \alpha \wedge \nu=0$ because $L_{Y} \alpha=0$ for all harmonic vector fields $Y$ on the 2-torus, $\alpha$ being a constant 1-form.

Hence on the 2-torus, for constant $\alpha$ and initial conditions $u_{0}$ Hamiltonian vector field and $f_{0}$ function with zero integral, $u$ will be Hamiltonian and $f$ will have zero integral at every time $t$. The Hamiltonian vector field is $H_{\psi}=\partial_{y} \psi \partial_{x}-\partial_{x} \psi \partial_{y}$ and the Poisson bracket $L_{H_{\psi}} f=\{f, \psi\}$ 
is the Jacobian of $f$ and $\psi$. If $\alpha=-\beta d y$ and $a=-1$ we get the equation for stream function $\psi$ and vorticity function $\omega=\Delta \psi$ :

$$
\begin{aligned}
& \partial_{t} \omega=-\{\omega, \psi\}-\beta \partial_{x} f, \\
& \partial_{t} f=-\{f, \psi\}+\beta \partial_{x} \psi .
\end{aligned}
$$

Let $\xi=g \frac{\rho-\rho_{0}}{\rho_{0}}$ be a buoyancy variable measuring the deviation of a density $\rho$ from a background value $\rho_{0}$, with $g$ the gravity acceleration. The background stratification $\rho_{0}$ is assumed to be exponential, characterized by the constant Brunt-Väisälä frequency $N=\left(-g \frac{d \log \rho_{0}}{d y}\right)^{\frac{1}{2}}$. The equation for a stratified fluid in Boussinesq approximation [61] is the geodesic equation (17.3) for $\beta=N$ constant and $f=N^{-1} \xi$ :

$$
\begin{aligned}
& \partial_{t} \omega=-\{\omega, \psi\}-\partial_{x} \xi, \\
& \partial_{t} \xi=-\{\xi, \psi\}+N^{2} \partial_{x} \psi .
\end{aligned}
$$

When the Brunt-Väisälä frequency $N$ is an integer and $\xi$ has zero integral (at time zero), then the stratified fluid equation is a geodesic equation on a Lie group [58].

\section{$18 H^{1}$ metrics}

\section{Camassa-Holm equation}

\section{The Camassa-Holm equation [8]}

$$
\partial_{t}\left(u-u^{\prime \prime}\right)=-3 u u^{\prime}+2 u^{\prime} u^{\prime \prime}+u u^{\prime \prime \prime}
$$

is the geodesic equation for the right invariant metric on $\operatorname{Diff}\left(S^{1}\right)$ given by the $H^{1}$ scalar product $\langle X, Y\rangle=\int_{S^{1}}\left(X Y+X^{\prime} Y^{\prime}\right) d x=\int_{S^{1}} X\left(1-\partial_{x}^{2}\right) Y d x[29]$.

Indeed, one gets from

$$
\begin{aligned}
\left\langle\operatorname{ad}(X)^{\top} Y, Z\right\rangle & =\left\langle Y, X^{\prime} Z-X Z^{\prime}\right\rangle=\int_{S^{1}}\left(Y\left(X^{\prime} Z-X Z^{\prime}\right)+Y^{\prime}\left(X^{\prime \prime} Z-X Z^{\prime \prime}\right)\right) d x \\
& =\int_{S^{1}} Z\left(2 Y X^{\prime}+Y^{\prime} X-2 Y^{\prime \prime} X^{\prime}-Y^{\prime \prime \prime} X\right) d x
\end{aligned}
$$

that $\operatorname{ad}(X)^{\top} Y=\left(1-\partial_{x}^{2}\right)^{-1}\left(2 Y X^{\prime}+Y^{\prime} X-2 Y^{\prime \prime} X^{\prime}-Y^{\prime \prime \prime} X\right)$. Plugging $\operatorname{ad}(X)^{\top} X=(1-$ $\left.\partial_{x}^{2}\right)^{-1}\left(3 X X^{\prime}-2 X^{\prime} X^{\prime \prime}-X X^{\prime \prime \prime}\right)$ into Euler's equation (2.1) one obtains the Camassa-Holm shallow water equation for $u: I \rightarrow C^{\infty}\left(S^{1}\right)$.

Since $m=A(u)=u-u^{\prime \prime}$, the Hamiltonian form of the Camassa-Holm equation is

$$
\partial_{t} m=-u m^{\prime}-2 u^{\prime} m \text {. }
$$

Remark 6. Considering the right invariant $H^{1}$ metric on the Bott-Virasoro group (5.2), an extended Camassa-Holm equation is obtained [38]

$$
\partial_{t}\left(u-u^{\prime \prime}\right)=-3 u u^{\prime}+2 u^{\prime} u^{\prime \prime}+u u^{\prime \prime \prime}-2 a u^{\prime \prime \prime}, \quad a \in \mathbb{R} .
$$

Indeed, the identity $\omega(X, Y)=\langle k(X), Y\rangle$ for the Virasoro cocycle $\omega(X, Y)=2 \int_{S^{1}} X^{\prime \prime \prime} Y d x$ and the $H^{1}$ scalar product implies $k(X)=2\left(1-\partial_{x}^{2}\right)^{-1} X^{\prime \prime \prime}$. Now by Corollary 2 the geodesic equation is the extended Camassa-Holm equation above.

The homogeneous manifold $\operatorname{Diff}\left(S^{1}\right) / S^{1}$ is a coadjoint orbit of the Bott-Virasoro group. The Hunter-Saxton equation describing weakly nonlinear unidirectional waves [22]

$$
\partial_{t} u^{\prime \prime}=-2 u^{\prime} u^{\prime \prime}-u u^{\prime \prime \prime}
$$

is a geodesic equation on $\operatorname{Diff}\left(S^{1}\right) / S^{1}$ with the right invariant metric defined by the scalar product $\langle X, Y\rangle=\int_{S^{1}} X^{\prime} Y^{\prime} d x[25]$. 


\section{Higher dimensional Camassa-Holm equation}

The higher dimensional Camassa-Holm equation (also called EPDiff or averaged template matching equation) $[19,20]$ is the geodesic equation for the right invariant $H^{1}$ metric

$$
\langle X, Y\rangle=\int_{M}\left(g(X, Y)+\alpha^{2} g(\nabla X, \nabla Y)\right) \mu,
$$

on $\operatorname{Diff}(M)$ for compact $M$. Because $\nabla^{*} \nabla=\Delta+$ Ric, this scalar product can be rewritten with the help of the rough Laplacian $\Delta_{R}=\Delta+\operatorname{Ric}$ as $\langle X, Y\rangle=\int_{M}\left(g\left(X-\alpha^{2} \Delta_{R} X, Y\right) \mu\right.$, so the momentum density of the fluid $m=A(u)$ is $m=\left(1-\alpha^{2} \Delta_{R}\right) u$. It follows that the adjoint of $\operatorname{ad}(X)$ with respect to (18.2) is conjugate by $1-\alpha^{2} \Delta_{R}$ to the adjoint of $\operatorname{ad}(X)$ with respect to the $L^{2}$ metric (3.1) computed to be (4.3). Hence $\left(1-\alpha^{2} \Delta_{R}\right) \operatorname{ad}(X)^{\top} Y=$ $(\nabla X)^{\top}\left(Y-\alpha^{2} \Delta_{R} Y\right)+\nabla_{X}\left(Y-\alpha^{2} \Delta_{R} Y\right)+(\operatorname{div} X)\left(Y-\alpha^{2} \Delta_{R} Y\right)$. We get as geodesic equation the higher dimensional Camassa-Holm equation

$$
\begin{aligned}
\partial_{t}\left(1-\alpha^{2} \Delta_{R}\right) u= & -u \operatorname{div} u+\alpha^{2}(\operatorname{div} u) \Delta_{R} u-\nabla_{u} u+\alpha^{2} \nabla_{u}\left(\Delta_{R} u\right) \\
& -(\nabla u)^{\top} u+\alpha^{2}(\nabla u)^{\top} \Delta_{R} u .
\end{aligned}
$$

In Hamiltonian form this equation is

$$
\partial_{t} m=-\nabla_{u} m-(\nabla u)^{\top} m-(\operatorname{div} u) m \quad \text { for } \quad m=A(u)=u-\alpha^{2} \Delta_{R} u .
$$

In particular for $M=S^{1}$ we get the Camassa-Holm equation (18.1).

When $M$ is a manifold with boundary and we put Neumann or mixed conditions on the boundary, then the $H^{1}$ scalar product (18.2) has to be replaced by

$$
\langle X, Y\rangle=\int_{M}\left(g(X, Y)+2 \alpha^{2} g(\operatorname{Def} X, \operatorname{Def} Y)\right) \mu
$$

where Def $X=\frac{1}{2}\left(\nabla X+(\nabla X)^{\top}\right)$ denotes the deformation $(1,1)$-tensor of $X[15]$.

\section{Averaged Euler equation}

For a compact Riemannian manifold $M$ we consider the right invariant metric on the group $\operatorname{Diff}_{\mu}(M)$ of volume preserving diffeomorphisms given by the $H^{1}$ scalar product (18.2) on vector fields. The geodesic equation is the (Lagrangian) averaged Euler equation [31, 50], also called LAE- $\alpha$ equation:

$$
\partial_{t}\left(1-\alpha^{2} \Delta_{R}\right) u=-\nabla_{u}\left(1-\alpha^{2} \Delta_{R}\right) u+\alpha^{2}(\nabla u)^{\top}\left(\Delta_{R} u\right)-\operatorname{grad} p .
$$

Indeed, from $\left\langle\operatorname{ad}(X)^{\top} Y, Z\right\rangle=\int_{M} g\left(Y-\alpha^{2} \Delta_{R} Y, \nabla_{Z} X-\nabla_{X} Z\right) \mu=\int_{M} g\left((\nabla X)^{\top}\left(Y-\alpha^{2} \Delta_{R} Y\right)+\right.$ $\left.\nabla_{X}\left(Y-\alpha^{2} \Delta_{R} Y\right), Z\right) \mu$ we obtain that

$$
\left(1-\alpha^{2} \Delta_{R}\right)\left(\operatorname{ad}(X)^{\top} Y\right)=P\left((\nabla X)^{\top} Y+\nabla_{X}\left(1-\alpha^{2} \Delta_{R}\right) Y-\alpha^{2}(\nabla X)^{\top}\left(\Delta_{R} Y\right)\right)
$$

and we use Euler's equation (2.1) to get (18.4). In Hamiltonian form this equation is

$$
\partial_{t} m=-\nabla_{u} m-(\nabla u)^{\top} m-\operatorname{grad} p
$$

for $m=A(u)=u-\alpha^{2} \Delta_{R} u$.

As in the higher dimensional Camassa-Holm equation, when Neumann or mixed conditions on the boundary of $M$ are imposed, one has to consider the $H^{1}$ scalar product (18.3). 


\section{Systems of two evolutionary equations}

From [12] we know that a basis for $H^{2}\left(\mathfrak{X}\left(S^{1}\right), C^{\infty}\left(S^{1}\right)\right)$ is represented by $\sigma(X, Y)=X^{\prime} Y-$ $X Y^{\prime}$ and the Virasoro cocycle $\omega(X, Y)=\int_{S^{1}}\left(X^{\prime} Y^{\prime \prime}-X^{\prime \prime} Y^{\prime}\right) d x \in \mathbb{R} \subset C^{\infty}\left(S^{1}\right)$; a basis for $H^{2}\left(\mathfrak{X}\left(S^{1}\right), \Omega^{1}\left(S^{1}\right)\right)$ is represented by the cocycles

$$
\sigma_{1}(X, Y)=X Y^{\prime \prime}-X^{\prime \prime} Y, \quad \omega_{1}(X, Y)=X^{\prime} Y^{\prime \prime}-X^{\prime \prime} Y^{\prime} ;
$$

a basis for $H^{2}\left(\mathfrak{X}\left(S^{1}\right), \Omega^{2}\left(S^{1}\right)\right)$ is represented by the cocycles

$$
\sigma_{2}(X, Y)=X^{\prime \prime \prime} Y-X Y^{\prime \prime \prime}, \quad \omega_{2}(X, Y)=X^{\prime \prime \prime} Y^{\prime}-X^{\prime} Y^{\prime \prime \prime} .
$$

Only the cocycles $\omega, \omega_{1}$ and $\omega_{2}$ (whose expressions involve only derivatives of $X$ and $Y$ ) integrate to group cocycles [45].

The Euler equations for the $L^{2}$ or $H^{1}$ scalar product on the corresponding Abelian extensions provide systems of two equations, generalizing Burgers (4.1) or Camassa-Holm (18.1) equation. We exemplify with the 2-cocycle $\sigma$ taking values in the module of functions on the circle. The Euler equations for the $L^{2}$ scalar product on $C^{\infty}\left(S^{1}\right) \rtimes \mathfrak{X}\left(S^{1}\right)$ and on $C^{\infty}\left(S^{1}\right) \rtimes_{\sigma} \mathfrak{X}\left(S^{1}\right)$ are

$$
\begin{aligned}
& \partial_{t} u=-3 u u^{\prime}-f f^{\prime}, \\
& \partial_{t} f=-u f^{\prime}-u^{\prime} f,
\end{aligned}
$$

and

$$
\begin{aligned}
& \partial_{t} u=-3 u u^{\prime}+u f^{\prime}+2 u^{\prime} f-f f^{\prime}, \\
& \partial_{t} f=-u f^{\prime}-u^{\prime} f .
\end{aligned}
$$

The Euler equations for the $H^{1}$ scalar product on $C^{\infty}\left(S^{1}\right) \rtimes \mathfrak{X}\left(S^{1}\right)$ and on $C^{\infty}\left(S^{1}\right) \rtimes_{\sigma} \mathfrak{X}\left(S^{1}\right)$ are

$$
\begin{aligned}
& \partial_{t}\left(u-u^{\prime \prime}\right)=-3 u u^{\prime}+2 u^{\prime} u^{\prime \prime}+u u^{\prime \prime \prime}-f f^{\prime}+f^{\prime} f^{\prime \prime \prime}, \\
& \partial_{t}\left(f-f^{\prime \prime}\right)=-u f^{\prime}-u^{\prime} f+u f^{\prime \prime \prime}+u^{\prime} f^{\prime \prime},
\end{aligned}
$$

and

$$
\begin{aligned}
& \partial_{t}\left(u-u^{\prime \prime}\right)=-3 u u^{\prime}+2 u^{\prime} u^{\prime \prime}+u u^{\prime \prime \prime}-2 u^{\prime} f-u f^{\prime}+2 u^{\prime} f^{\prime \prime}+u f^{\prime \prime \prime}-f f^{\prime}+f^{\prime} f^{\prime \prime \prime}, \\
& \partial_{t}\left(f-f^{\prime \prime}\right)=-u f^{\prime}-u^{\prime} f+u f^{\prime \prime \prime}+u^{\prime} f^{\prime \prime}
\end{aligned}
$$

One can consider central extensions of semidirect products of $\mathfrak{X}\left(S^{1}\right)$ with modules of densities as in Remark 5 [45]. For instance the 1-cocycle $\alpha(X)=X^{\prime \prime}$ on $\mathfrak{X}\left(S^{1}\right)$ with values in $\Omega^{1}\left(S^{1}\right)$, the module dual to $C^{\infty}\left(S^{1}\right)$, gives the 2-cocycle $\omega\left(\left(f_{1}, X_{1}\right),\left(f_{2}, X_{2}\right)\right)=\int_{S^{1}}\left(X_{1}^{\prime \prime} f_{2}-X_{2}^{\prime \prime} f_{1}\right) d x$ on the semidirect product $C^{\infty}\left(S^{1}\right) \rtimes \mathfrak{X}\left(S^{1}\right)$. The geodesic equation for the $L^{2}$ scalar product on the central extension $\left(C^{\infty}\left(S^{1}\right) \rtimes \mathfrak{X}\left(S^{1}\right)\right) \times_{\omega} \mathbb{R}$ is

$$
\begin{aligned}
& \partial_{t} u=-3 u u^{\prime}-f f^{\prime}-a f^{\prime \prime}, \\
& \partial_{t} f=-u f^{\prime}-u^{\prime} f-a u^{\prime \prime}, \quad a \in \mathbb{R} .
\end{aligned}
$$

\section{Conclusions}

This survey article presents the formal deduction as geodesic equations on diffeomorphism groups with right invariant metrics of several PDE's of hydrodynamical type. Sometimes extensions of diffeomorphism groups by central or Abelian sugroups come into play and the corresponding Lie algebra 2-cocycles introduce additional terms to the geodesic equations.

These equations are Hamiltonian equations too, possessing rich geometric structures. Some of them are completely integrable. But presenting these results is beyond the scope of this article. 


\section{Acknowledgements}

This work was done with the financial support of Romanian Ministery of Education and Research under the grant CNCSIS 95GR/2007. I acknowledge the support from the ICTP Office of External Activities for attending the Seventh International Conference "Symmetry in Nonlinear Mathematical Physics" in Kyiv.

I am most grateful to Tudor Ratiu and Francois Gay-Balmaz for their preprints and for very good suggestions, and to the referees for their very substantial and constructive comments.

\section{References}

[1] Alekseevsky A., Michor P.W., Ruppert W., Extensions of super Lie algebras, J. Lie Theory 15 (2005), 125-134, math.QA/0101190.

[2] Arnold V.I., Sur la géométrie differentielle des groupes de Lie de dimension infinie et ses applications à l'hydrodynamique des fluides parfaits, Ann. Inst. Fourier (Grenoble) 16 (1966), 319-361.

[3] Arnold V.I., Khesin B.A., Topological methods in hydrodynamics, Springer, Berlin, 1998.

[4] Bao D., Ratiu T., On the geometrical origin of a degenerate Monge-Ampère equation, Proc. Sympos. Pure Math. 54 (1993), 55-68.

[5] Billig Y., Magnetic hydrodynamics with asymmetric stress tensor, J. Math. Phys. 46 (2005), 043101, 13 pages, math-ph/0401052.

[6] Brenier Y., Minimal geodesics on groups of volume-preserving maps and generalized solutions of the Euler equations, Comm. Pure Appl. Math. 52 (1999), 411-452.

[7] Burgers J., A mathematical model illustrating the theory of turbulence, Adv. Appl. Mech. 1 (1948), $171-199$.

[8] Camassa R., Holm D., An integrable shallow water equation with peaked solitons, Phys. Rev. Lett. 71 (1993), 1661-1664, patt-sol/9305002.

[9] Constantin A., Kappeler T., Kolev B., Topalov P., On geodesic exponential maps of the Virasoro group, Ann. Global Anal. Geom. 31 (2007), 155-180.

[10] Ebin D., Marsden J., Groups of diffeomorphisms and the motion of an incompressible fluid, Ann. of Math. (2) 92 (1970), 102-163.

[11] Eliashberg Ya., Ratiu T., The diameter of the symplectomorphism group is infinite, Invent. Math. 103 (1991), 327-340.

[12] Fuks D.B., Cohomology of infinite-dimensional lie algebras, Contemp. Sov. Math., Consultants Bureau, New York, 1986.

[13] Gay-Balmaz F., Ratiu T., The Lie-Poisson structure of the LAE- $\alpha$ equation, Dyn. Partial Differ. Equ. 2 (2005), 25-57, math.DG/0504381.

[14] Gay-Balmaz F., Ratiu T., Euler-Poincaré and Lie-Poisson formulations of Euler-Yang-Mills equations, Preprint, 2006.

[15] Gay-Balmaz F., Ratiu T., Well posedness of higher dimensional Camassa-Holm equations on manifolds, Preprint, 2007.

[16] Haller S., Teichmann J., Vizman C., Totally geodesic subgroups of diffeomorphisms, J. Geom. Phys. 42 (2002), 342-354, math.DG/0103220.

[17] Hattori Y., Ideal magnetohydrodynamics and passive scalar motion as geodesics on semidirect product groups, J. Phys. A: Math. Gen. 27 (1994), L21-L25.

[18] Hirani A.N., Marsden J.E., Arvo J., Averaged template matching equations, Lecture Notes Computer Science, Vol. 2134, Proc. Third Int. Workshop EMMCVPR, 2001, Springer, Berlin-Heidelberg, 528-543.

[19] Holm D., Marsden J., Ratiu T., The Euler-Poincaré equations and semidirect products with applications to continuum theories, Adv. Math. 137 (1997), 1-81, chao-dyn/9801015.

[20] Holm D., Marsden J., Momentum maps and measure-valued solutions (peakons, filaments and sheets) for the EPDiff equation, in Festschrift for Alan Weinstein, Birkhäuser, Boston, 2003, 203-235, nlin.CD/0312048.

[21] Holm D., Zeitlin V., Hamilton's principle for quasigeostrophic motion, Phys. Fluids 10 (1998), 800-806, chao-dyn/9801018. 
[22] Hunter J.K., Saxton R., Dynamics of director fields, SIAM J. Appl. Math. 51 (1991), 1498-1521.

[23] Ismagilov R.S., Representations of infinite-dimensional groups, AMS Translations of Mathematical Monographs, Vol. 152, American Mathematical Society, Providence, RI, 1996.

[24] Ismagilov R.S., Inductive limits of area-preserving diffeomorphism groups, Funct. Anal. Appl. 37 (2003), 191-202.

[25] Khesin B., Misiolek G., Euler equations on homogeneous spaces and Virasoro orbits, Adv. Math. 176 (2003), 116-144, math.SG/0210397.

[26] Khesin B., Misiolek G., Asymptotic directions, Monge-Ampère equations and the geometry of diffeomorphism groups, J. Math. Fluid. Mech. 7 (2005), S365-S375, math.DG/0504556.

[27] Kirillov A.A., The orbit method. II. Infinite dimensional Lie groups and Lie algebras, Contemp. Math. 145 (1993), 33-63.

[28] Kriegl A., Michor P.W., The convenient setting of global analysis, Mathematical Surveys and Monographs, Vol. 53, Amer. Math. Soc., Providence, RI, 1997.

[29] Kouranbaeva S., The Camassa-Holm equation as a geodesic flow on the diffeomorphism group, J. Math. Phys. 40 (1999), 857-868, math-ph/9807021.

[30] Marsden J., Ratiu T., Introduction to mechanics and symmetry, 2nd ed., Springer, 1999.

[31] Marsden J., Ratiu T., Shkoller S., The geometry and analysis of the averaged Euler equations and a new diffeomorphism group, Geom. Funct. Anal. 10 (2000), 582-599, math.AP/9908103.

[32] Marsden J.E., Ratiu T., Weinstein A., Semidirect product and reduction in mechanics, Trans. Amer. Math. Soc. 281 (1984), 147-177.

[33] Michor P.W., Mumford D., Vanishing geodesic distance on spaces of submanifolds and diffeomorphisms, Doc. Math. 10 (2005), 217-245, math.DG/0409303.

[34] Michor P.W., Ratiu T., On the geometry of the Virasoro-Bott group, J. Lie Theory 8 (1998), 293-309, math.DG/9801115.

[35] Milnor J., Remarks on infinite-dimensional Lie groups, in Proc. Summer School on Quantum Gravity, Editor B. DeWitt, North Holland, Amsterdam, 1984, 1007-1057.

[36] Misiolek G., Stability of flows of ideal fluids and the geometry of the group of diffeomorphisms, Indiana Univ. Math. J. 42 (1993), 215-235.

[37] Misiolek G., Conjugate points in the Bott-Virasoro group and the KdV equation, Proc. Amer. Math. Soc. 125 (1997), 935-940.

[38] Misiolek G., A shallow water equation as a geodesic flow on the Bott-Virasoro group, J. Geom. Phys. 24 (1998), 203-208.

[39] Misiolek G., Classical solutions of the periodic Camassa-Holm equation, Geom. Funct. Anal. 12 (2002), 1080-1104.

[40] Misiolek G., Conjugate points in $\operatorname{SDiff}\left(T^{2}\right)$, Proc. Amer. Math. Soc. 124 (1996), 977-982.

[41] Moreau J.J., Une méthode de "cinématique fonctionnelle" en hydrodynamique, C. R. Acad. Sci. Paris 249 (1959), 2156-2158.

[42] Nakamura F., Hattori Y., Kambe T., Geodesics and curvature of a group of diffeomorphisms and motion of an ideal fluid, J. Phys. A: Math. Gen. 25 (1992), L45-L50.

[43] Neeb K.-H., Abelian extensions of infinite-dimensional Lie groups, Travaux Math. XV (2004), 69-194, math.GR/0402303.

[44] Ovsienko V.Y., Khesin B.A., Korteweg-de Vries superequations as an Euler equation, Funct. Anal. Appl. 21 (1987), 329-331.

[45] Ovsienko V., Roger C., Generalizations of Virasoro group and Virasoro algebra through extensions by modules of tensor densities on $S^{1}$, Indag. Math. (N.S.) 9 (1998), 277-288.

[46] Pekarsky S., Shkoller S., On the stability of periodic 2D Euler- $\alpha$ flows, math.AP/0007050.

[47] Poincaré H., Sur une nouvelle forme des équations de la méchanique, C. R. Acad. Sci. 132 (1901), 369-371.

[48] Preston S.C., For ideal fluids, Eulerian and Lagrangian instabilities are equivalent, Geom. Funct. Anal. 14 (2004), 1044-1062.

[49] Roger C., Extensions centrales d'algèbres et de groupes de Lie de dimension infinie, algèbre de Virasoro et généralisations, Rep. Math. Phys. 35 (1995), 225-266. 
[50] Shkoller S., Geometry and curvature of diffeomorphism groups with $H^{1}$ metric and mean hydrodynamics, J. Funct. Anal. 160 (1998), 337-365, math.AP/9807078.

[51] Shkoller S., Analysis on groups of diffeomorphisms of manifolds with boundary and the averaged motion of a fluid, J. Differential Geom. 55 (2000), 145-191.

[52] Shnirel'man A.I., The geometry of the group of diffeomorphisms and the dynamics of an incompressible fluid, Math. Sb. 56 (1987), 79-105.

[53] Shnirel'man A.I., Generalized fluid flows, their approximation and applications, Geom. Funct. Anal. 4 (1994), 586-620.

[54] Vishik S.M., Dolzhanskii F.V., Analogs of the Euler-Lagrange equations and magnetohydrodynamics equations related to Lie groups, Sov. Math. Doklady 19 (1978), 149-153.

[55] Vizman C., Curvature and geodesics on diffeomorphism groups, in Proceedings of the Fourth International Workshop on Differential Geometry, Braşov, Romania, 1999, 298-305.

[56] Vizman C., Geodesics and curvature of semidirect product groups, Rend. Circ. Mat. Palermo (2) Suppl. 66 (2001), 199-206, math.DG/0103141.

[57] Vizman C., Geodesics on extensions of Lie groups and stability: the superconductivity equation, Phys. Lett. A 284 (2001), 23-30, math.DG/0103140.

[58] Vizman C., Central extensions of semidirect products and geodesic equations, Phys. Lett. A 330 (2004), 460-469.

[59] Vizman C., Central extensions of the Lie algebra of symplectic vector fields, J. Lie Theory 16 (2005), 297-309.

[60] Vizman C., Quasigeostrophic motion, stream functions and cocycles, J. Nonlinear Math. Phys., to appear.

[61] Zeitlin V., Vorticity and waves: geometry of phase-space and the problem of normal variables, Phys. Lett. A 164 (1992), 177-183.

[62] Zeitlin V., Pasmanter R.A., On the differential geometric approach to geophysical flows, Phys. Lett. A 189 (1994), 59-63.

[63] Zeitlin V., Kambe T., Two-dimensional ideal magnetohydrodynamics and differential geometry, J. Phys. A: Math. Gen. 26 (1993), 5025-5031. 